\title{
Neimark-Sacker Bifurcation and Chaos Control in a Fractional-Order Plant-Herbivore Model
}

\author{
Qamar Din, ${ }^{1}$ A. A. Elsadany, ${ }^{2}$ and Hammad Khalil ${ }^{3}$ \\ ${ }^{1}$ Department of Mathematics, The University of Poonch Rawalakot, Rawalakot 12350, Pakistan \\ ${ }^{2}$ Basic Science Department, Faculty of Computers and Informatics, Suez Canal University, New Campus, Ismailia 41522, Egypt \\ ${ }^{3}$ Department of Mathematics, University of Education, Attock Campus, Lahore, Punjab, Pakistan
}

Correspondence should be addressed to Qamar Din; qamar.sms@gmail.com

Received 14 November 2016; Accepted 27 February 2017; Published 10 April 2017

Academic Editor: Josef Diblik

Copyright () 2017 Qamar Din et al. This is an open access article distributed under the Creative Commons Attribution License, which permits unrestricted use, distribution, and reproduction in any medium, provided the original work is properly cited.

\begin{abstract}
This work is related to dynamics of a discrete-time 3-dimensional plant-herbivore model. We investigate existence and uniqueness of positive equilibrium and parametric conditions for local asymptotic stability of positive equilibrium point of this model. Moreover, it is also proved that the system undergoes Neimark-Sacker bifurcation for positive equilibrium with the help of an explicit criterion for Neimark-Sacker bifurcation. The chaos control in the model is discussed through implementation of two feedback control strategies, that is, pole-placement technique and hybrid control methodology. Finally, numerical simulations are provided to illustrate theoretical results. These results of numerical simulations demonstrate chaotic long-term behavior over a broad range of parameters. The computation of the maximum Lyapunov exponents confirms the presence of chaotic behavior in the model.
\end{abstract}

\section{Introduction and Preliminaries}

In [1], authors proposed a mathematical model governed by ordinary differential equations related to the interaction between a plant and an insect. We extend this model by interchanging ordinary differential equations into fractionalorder differential equations. Arguing as in [1], we assume that the larval stage of an insect develops and grows at the expense of nonreproductive tissues of the plant; on the other hand, these insects at their adult stage convey pollen to or deposit pollen on the flowers of the same plant, so allowing fertilization. Moreover, we denote $P, L, A$, and $F$ as densities of plant species, larval species of insect, adult species of insect, and flower species involving in pollination, respectively. The interaction among $P, L, A$, and $F$ is described by the following system of nonlinear differential equations:

$$
\begin{aligned}
& \frac{d P}{d \tau}=a P(1-b P)+c d F A-a_{1} P L, \\
& \frac{d F}{d \tau}=b_{1} P-c_{1} F-d F A,
\end{aligned}
$$

$$
\begin{aligned}
& \frac{d L}{d \tau}=d_{1} F A+a_{2} A-b_{2} a_{1} P L-c_{2} L, \\
& \frac{d A}{d \tau}=b_{2} a_{1} P L-d_{2} A .
\end{aligned}
$$

Then, we recall the definition of Caputo fractional-order derivative [2] for any function $f$, and it is given by

$$
D^{\alpha} f(t)=I^{m-\alpha} f^{(m)}(t), \quad \alpha>0,
$$

where $m$ is the least positive integer satisfying $m \geq \alpha$ and $I^{9}$ denotes integral operator of Riemann-Liouville type with order $\vartheta$, and it is defined by

$$
I^{\vartheta} g(t)=\frac{1}{\Gamma(\vartheta)} \int_{0}^{t}(t-s)^{\vartheta-1} g(s) d s, \quad \vartheta>0,
$$


where $\Gamma(\cdot)$ represents Euler's Gamma function. Furthermore, the fractional-order counterpart of (5) is given by the following system:

$$
\begin{aligned}
& \frac{d^{\alpha} P}{d \tau^{\alpha}}=a P(1-b P)+c d F A-a_{1} P L, \\
& \frac{d^{\alpha} F}{d \tau^{\alpha}}=b_{1} P-c_{1} F-d F A, \\
& \frac{d^{\alpha} L}{d \tau^{\alpha}}=d_{1} F A+a_{2} A-b_{2} a_{1} P L-c_{2} L, \\
& \frac{d^{\alpha} A}{d \tau^{\alpha}}=b_{2} a_{1} P L-d_{2} A,
\end{aligned}
$$

where $0<\alpha \leq 1, d^{\alpha} / d \tau^{\alpha}$ is in the sense of the Caputo fractional derivative defined in (2), $a$ is plant intrinsic growth rate, $b$ is plant intraspecific self-regulation coefficient (also the inverse is its carrying capacity), $d$ denotes pollination rate, $a_{1}$ is called herbivory rate, $b_{1}$ is flower production rate, $c_{1}$ is flower decay rate, $c_{2}$ and $d_{2}$ are larva and adult mortality rates, $c$ is plant pollination efficiency ratio, $d_{1}$ denotes adult consumption efficiency ratio, and $b_{2}$ is called the maturation rate for brevity. Moreover, parameter $a_{2}$ represents a reproduction rate resulting from the pollination of other plants species. We now consider the fact that flowers last for a very short time as compared to the life cycles of plants and insects. This means that the variables $P, L$, and $A$ have slower dynamics, and, on the other hand, the variable $F$ has fast dynamics [3]. In case of steady states of plants and insects, one can find a steady state of flowers by putting right hand side of second equation of system (4) that is equal to zero, that is, $b_{1} P-c_{1} F-d F A=0$; then it follows that $F=b_{1} P /\left(c_{1}+d A\right)$ [1]. Putting $F=b_{1} P /\left(c_{1}+d A\right)$ in system (4), we obtain the following 3 -dimensional fractional-order system:

$$
\begin{aligned}
& \frac{d^{\alpha} P}{d \tau^{\alpha}}=a P(1-b P)+\frac{d b_{1} c A P}{c_{1}+d A}-a_{1} P L, \\
& \frac{d^{\alpha} L}{d \tau^{\alpha}}=\frac{d_{1} b_{1} A P}{c_{1}+d A}+a_{2} A-b_{2} a_{1} P L-c_{2} L, \\
& \frac{d^{\alpha} A}{d \tau^{\alpha}}=b_{2} a_{1} P L-d_{2} A .
\end{aligned}
$$

For lenient mathematical analysis, one can reduce the number of parameters in system (5) by using the following transformations:

$$
\begin{aligned}
& x=b P, \\
& y=b L, \\
& z=b A, \\
& t=a \tau .
\end{aligned}
$$

We have the following dimensionless system:

$$
\begin{aligned}
& \frac{d^{\alpha} x}{d t^{\alpha}}=x(1-x)+\frac{\lambda c x z}{\eta+z}-\beta x y, \\
& \frac{d^{\alpha} y}{d t^{\alpha}}=\frac{\lambda d_{1} x z}{\eta+z}+\phi z-b_{2} \beta x y-\mu y, \\
& \frac{d^{\alpha} z}{d t^{\alpha}}=\beta x y-v z,
\end{aligned}
$$

where $\lambda=b_{1} / a, \eta=c_{1} b / d, \beta=a_{1} / a b, \mu=c_{2} / a, v=d_{2} / a$, and $\phi=a_{2} / a$. Since population densities cannot be negative, the state space of system (7) is given by

$$
\left\{(x, y, z) \in \mathbb{R}^{3}: x \geq 0, y \geq 0, z \geq 0\right\} .
$$

Due to efficient computational results, discrete dynamical systems are much better than related systems in differential equations. Particularly, in case of nonoverlapping generations, difference equations are more suitable to study the behavior of population models [4-8]. For more details on some interesting population models both in differential equations and in difference equations, we refer the interested reader to [9-12]. It is very interesting to investigate the parametric conditions for existence of Neimark-Sacker bifurcation and to discuss chaos control techniques due to emergence of Neimark-Sacker bifurcation for discrete-time population models. For some interesting results related to Neimark-Sacker bifurcation and chaos control of discretetime population models, we refer the reader to [13-17].

Now we consider the counterpart of (7) with piecewise constant arguments as follows:

$$
\begin{aligned}
\frac{d^{\alpha} x}{d t^{\alpha}}= & x([t / k] k)(1-x([t / k] k)) \\
& +\frac{\lambda c x([t / k] k) z([t / k] k)}{\eta+z([t / k] k)} \\
& -\beta x([t / k] k) y([t / k] k), \\
\frac{d^{\alpha} y}{d t^{\alpha}}= & \frac{\lambda d_{1} x([t / k] k) z([t / k] k)}{\eta+z([t / k] k)}+\phi z([t / k] k) \\
& -b_{2} \beta x([t / k] k) y([t / k] k)-\mu y([t / k] k), \\
\frac{d^{\alpha} z}{d t^{\alpha}}= & \beta x([t / k] k) y([t / k] k)-v z([t / k] k),
\end{aligned}
$$

with initial conditions $x(0)=x_{0}, y(0)=y_{0}, z(0)=z_{0}$. Furthermore, assume that $t \in[0, k)$; then it follows that $t / k \in$ $[0,1)$. So for $t \in[0, k)$, system (9) gives

$$
\begin{aligned}
& \frac{d^{\alpha} x}{d t^{\alpha}}=x_{0}\left(1-x_{0}\right)+\frac{\lambda c x_{0} z_{0}}{\eta+z_{0}}-\beta x_{0} y_{0}, \\
& \frac{d^{\alpha} y}{d t^{\alpha}}=\frac{\lambda d_{1} x_{0} z_{0}}{\eta+z_{0}}+\phi z_{0}-b_{2} \beta x_{0} y_{0}-\mu y_{0}, \\
& \frac{d^{\alpha} z}{d t^{\alpha}}=\beta x_{0} y_{0}-\nu z_{0} .
\end{aligned}
$$


The solution of (9) is given by

$$
\begin{aligned}
& x_{1}(t)=x_{0}+I^{\alpha}\left[x_{0}\left(1-x_{0}\right)+\frac{\lambda c x_{0} z_{0}}{\eta+z_{0}}-\beta x_{0} y_{0}\right], \\
& y_{1}(t)=y_{0}+I^{\alpha}\left[\frac{\lambda d_{1} x_{0} z_{0}}{\eta+z_{0}}+\phi z_{0}-b_{2} \beta x_{0} y_{0}-\mu y_{0}\right], \\
& z_{1}(t)=z_{0}+I^{\alpha}\left[\beta x_{0} y_{0}-v z_{0}\right]
\end{aligned}
$$

where $I^{\alpha}$ is the Riemann-Liouville integral operator of order $\alpha$ which is defined in (3). From (3) and (11), it follows that

$$
\begin{aligned}
& x_{1}(t) \\
& =x_{0} \\
& +\frac{t^{\alpha}}{\Gamma(\alpha+1)}\left[x_{0}\left(1-x_{0}\right)+\frac{\lambda c x_{0} z_{0}}{\eta+z_{0}}-\beta x_{0} y_{0}\right], \\
& y_{1}(t) \\
& =y_{0} \\
& +\frac{t^{\alpha}}{\Gamma(\alpha+1)}\left[\frac{\lambda d_{1} x_{0} z_{0}}{\eta+z_{0}}+\phi z_{0}-b_{2} \beta x_{0} y_{0}-\mu y_{0}\right], \\
& z_{1}(t)=z_{0}+\frac{t^{\alpha}}{\Gamma(\alpha+1)}\left[\beta x_{0} y_{0}-v z_{0}\right] \text {. }
\end{aligned}
$$

Similarly, for $t \in[k, 2 k)$, so that $t / k \in[1,2)$, we obtain

$$
\begin{aligned}
& \frac{d^{\alpha} x}{d t^{\alpha}}=x_{1}\left(1-x_{1}\right)+\frac{\lambda c x_{1} z_{1}}{\eta+z_{1}}-\beta x_{1} y_{1}, \\
& \frac{d^{\alpha} y}{d t^{\alpha}}=\frac{\lambda d_{1} x_{1} z_{1}}{\eta+z_{1}}+\phi z_{1}-b_{2} \beta x_{1} y_{1}-\mu y_{1}, \\
& \frac{d^{\alpha} z}{d t^{\alpha}}=\beta x_{1} y_{1}-v z_{1} .
\end{aligned}
$$

The solution of (13) is given by

$$
\begin{aligned}
& x_{2}(t)=x_{1}(k)+\frac{(t-k)^{\alpha}}{\Gamma(\alpha+1)}\left[x_{1}(k)\left(1-x_{1}(k)\right)\right. \\
& \left.+\frac{\lambda c x_{1}(k) z_{1}(k)}{\eta+z_{1}(k)}-\beta x_{1}(k) y_{1}(k)\right], \\
& y_{2}(t)=y_{1}(k)+\frac{(t-k)^{\alpha}}{\Gamma(\alpha+1)}\left[\frac{\lambda d_{1} x_{1}(k) z_{1}(k)}{\eta+z_{1}(k)}\right. \\
& \left.+\phi z_{1}(k)-b_{2} \beta x_{1}(k) y_{1}(k)-\mu y_{1}(k)\right], \\
& z_{2}(t)=z_{1}(k)+\frac{(t-k)^{\alpha}}{\Gamma(\alpha+1)}\left[\beta x_{1}(k) y_{1}(k)-v z_{1}(k)\right] .
\end{aligned}
$$

Repeating the above process $n$-times, the solution of (9) for $t \in[n k,(n+1) k)$ is given by

$$
\begin{aligned}
& x_{n+1}(t)=x_{n}(n k)+\frac{(t-n k)^{\alpha}}{\Gamma(\alpha+1)}\left[x_{n}(n k)\left(1-x_{n}(n k)\right)\right. \\
& \left.+\frac{\lambda c x_{n}(n k) z_{n}(n k)}{\eta+z_{n}(n k)}-\beta x_{n}(n k) y_{n}(n k)\right], \\
& y_{n+1}(t)=y_{n}(n k)+\frac{(t-n k)^{\alpha}}{\Gamma(\alpha+1)}\left[\frac{\lambda d_{1} x_{n}(n k) z_{n}(n k)}{\eta+z_{n}(n k)}\right. \\
& \left.+\phi z_{n}(n k)-b_{2} \beta x_{n}(n k) y_{n}(n k)-\mu y_{n}(n k)\right], \\
& z_{n+1}(t)=z_{n}(n k)+\frac{(t-n k)^{\alpha}}{\Gamma(\alpha+1)}\left[\beta x_{n}(n k) y_{n}(n k)\right. \\
& \left.-v z_{n}(n k)\right] .
\end{aligned}
$$

Next taking $t \rightarrow(n+1) k$ and adopting the notation of difference equations, system (15) yields

$$
\begin{aligned}
& x_{n+1} \\
& =x_{n} \\
& +\frac{k^{\alpha}}{\Gamma(\alpha+1)}\left[x_{n}\left(1-x_{n}\right)+\frac{\lambda c x_{n} z_{n}}{\eta+z_{n}}-\beta x_{n} y_{n}\right] \text {, } \\
& y_{n+1} \\
& =y_{n} \\
& +\frac{k^{\alpha}}{\Gamma(\alpha+1)}\left[\frac{\lambda d_{1} x_{n} z_{n}}{\eta+z_{n}}+\phi z_{n}-b_{2} \beta x_{n} y_{n}-\mu y_{n}\right], \\
& z_{n+1}=z_{n}+\frac{k^{\alpha}}{\Gamma(\alpha+1)}\left[\beta x_{n} y_{n}-v z_{n}\right]
\end{aligned}
$$

Our aim in this paper is to study the local asymptotic stability of equilibrium points of system (16). Moreover, NeimarkSacker bifurcation for positive equilibrium of system (16) is also investigated. In order to control the chaos due to emergence of Neimark-Sacker bifurcation, pole-placement and hybrid control strategies are implemented on system (16). Similar methods of discretization for fractional-order systems are also used in [18-22].

\section{Linearized Stability of System (16)}

First, we consider possible steady-state (equilibrium point) $\left(x^{*}, y^{*}, z^{*}\right)$ of system (16), which can be obtained by solving the following system: 


$$
\begin{aligned}
x^{*}\left(1-x^{*}\right)+\frac{\lambda c x^{*} z^{*}}{\eta+z^{*}}-\beta x^{*} y^{*} & =0, \\
\frac{\lambda d_{1} x^{*} z^{*}}{\eta+z^{*}}+\phi z^{*}-b_{2} \beta x^{*} y^{*}-\mu y^{*} & =0, \\
\beta x^{*} y^{*}-v z^{*} & =0 .
\end{aligned}
$$

Then it is easy to see that $\left(x^{*}, y^{*}, z^{*}\right)=(0,0,0)$ and $\left(x^{*}, y^{*}\right.$, $\left.z^{*}\right)=(1,0,0)$ are two solutions of system (17). It follows that $P_{0}=(0,0,0)$ and $P_{1}=(1,0,0)$ are two equilibria of system

(16). Neglecting the trivial equilibrium, we are left with

$$
\begin{aligned}
1-x^{*}+\frac{\lambda c z^{*}}{\eta+z^{*}}-\beta y^{*} & =0, \\
\frac{\lambda d_{1} x^{*} z^{*}}{\eta+z^{*}}+\phi z^{*}-b_{2} \beta x^{*} y^{*}-\mu y^{*} & =0, \\
\beta x^{*} y^{*}-v z^{*} & =0 .
\end{aligned}
$$

It must be noted that the equilibrium point of system (16), that is, the solution of system (18), may not be unique, but we do not care how many. For biological reasons, we are only interested in positive solutions of (18). From system (18), we obtain

$$
\begin{aligned}
& y^{*}=\frac{\lambda d_{1} \beta \nu\left(x^{*}\right)^{2}+\phi \beta \eta \nu x^{*}-\eta v^{2}\left(b_{2} \beta x^{*}+\mu\right)}{\beta \nu x^{*}\left(b_{2} \beta x^{*}+\mu\right)-\phi \beta^{2}\left(x^{*}\right)^{2}}, \\
& z^{*}=\frac{\lambda d_{1} \beta\left(x^{*}\right)^{2}+\phi \beta \eta x^{*}-\eta \nu\left(b_{2} \beta x^{*}+\mu\right)}{\nu\left(b_{2} \beta x^{*}+\mu\right)-\phi \beta x^{*}},
\end{aligned}
$$

where $x^{*}$ is one of the roots of the following quartic polynomial:

$$
P(t)=A t^{4}+B t^{3}+C t^{2}+D t+E,
$$

such that

$$
\begin{aligned}
& A=\beta^{2} d_{1}\left(b_{2} \nu-\phi\right) \\
& B=\beta d_{1}\left(\beta c \lambda \phi+\beta d_{1} \lambda \nu+\beta \phi+\mu \nu-b_{2} \beta c \lambda \nu-b_{2} \beta \nu\right)
\end{aligned}
$$

$$
J(x, y, z)=\left[\begin{array}{c}
1+\frac{k^{\alpha}(1-2 x-y \beta+c z \lambda /(z+\eta))}{\Gamma(1+\alpha)} \\
\frac{k^{\alpha}\left(-y \beta b_{2}+z \lambda d_{1} /(z+\eta)\right)}{\Gamma(1+\alpha)} \\
\frac{k^{\alpha} y \beta}{\Gamma(1+\alpha)}
\end{array}\right.
$$

$$
\begin{aligned}
C & =\beta\left(\beta b_{2}^{2} c \eta \nu^{2}+\beta c \eta \phi^{2}+\beta d_{1} \eta \nu \phi-2 b_{2} \beta c \eta \nu \phi\right. \\
& \left.-b_{2} \beta d_{1} \eta \nu^{2}-c d_{1} \lambda \mu \nu-d_{1} \mu \nu\right), \\
D & =\beta \mu \nu \eta\left(2 b_{2} c \nu-2 c \phi-d_{1} \nu\right), \\
E & =c \eta \mu^{2} \nu^{2} .
\end{aligned}
$$

We are looking for the unique positive equilibrium point of system (18); for this, we have the following Descartes's rule of signs.

Lemma 1 (see [23]). Let $f(x)=a_{n} x^{n}+a_{n-1} x^{n-1}+\cdots+$ $a_{1} x+a_{0}$ be a polynomial function with real coefficients. Then the number of positive real roots of $f$ is either the same as the number of sign changes of $f(x)$ or less than the number of sign changes of $f(x)$ by a positive even integer. Moreover, if $f(x)$ has only one variation in sign, then $f$ has exactly one positive real root.

Using Lemma 1, we have the following result for existence of unique positive real root of polynomial $P(t)$ given in (20).

Lemma 2. Polynomial $P(t)$ in (20) has unique positive real root if one of the following conditions hold:

(i) $A<0, B<0, C<0, D<0, E>0$.

(ii) $A<0, B>0, C>0, D>0, E>0$.

Due to above analysis, we have the following result about the existence and uniqueness of positive equilibrium point of system (16).

Lemma 3. Under the conditions of Lemma 2, system (16) has unique positive equilibrium point if the following condition holds:

$$
\begin{aligned}
& \frac{\eta \nu b_{2}-\eta \phi}{2 \lambda d_{1}} \\
& +\frac{1}{2} \sqrt{\frac{\beta \eta^{2} \phi^{2}-2 \beta \eta^{2} \nu \phi b_{2}+\beta \eta^{2} \nu^{2} b_{2}^{2}+4 \eta \lambda \mu \nu d_{1}}{\beta \lambda^{2} d_{1}^{2}}} \\
& \quad<x^{*}<\frac{\mu \nu}{\beta \phi-\beta \nu b_{2}},
\end{aligned}
$$

where $x^{*}$ is unique positive real root of polynomial (20).

Next, the Jacobian matrix for system (16) evaluated at $(x, y, z)$ is given by

$$
\left.\begin{array}{cc}
-\frac{k^{\alpha} x \beta}{\Gamma(1+\alpha)} & \frac{c k^{\alpha} x \eta \lambda}{(z+\eta)^{2} \Gamma(1+\alpha)} \\
1+\frac{k^{\alpha}\left(-\mu-x \beta b_{2}\right)}{\Gamma(1+\alpha)} & \frac{k^{\alpha}\left((z+\eta)^{2} \phi+x \eta \lambda d_{1}\right)}{(z+\eta)^{2} \Gamma(1+\alpha)} \\
\frac{k^{\alpha} x \beta}{\Gamma(1+\alpha)} & 1-\frac{k^{\alpha} \nu}{\Gamma(1+\alpha)}
\end{array}\right]
$$


Theorem 4. For system (16), the following statements hold true:

(i) The trivial equilibrium point $P_{0}$ is unstable.

(ii) The equilibrium point $P_{1}$ is locally asymptotically stable if and only if $s^{\alpha} / \Gamma(1+\alpha)<2$ and

$$
\begin{aligned}
& k^{\alpha}\left(\sqrt{\eta}\left(\mu+\nu+\beta b_{2}\right)\right. \\
& \left.\quad+\sqrt{\eta\left((\mu-\nu)^{2}+4 \beta \phi+\beta b_{2}\left(2 \mu-2 \nu+\beta b_{2}\right)\right)+4 \beta \lambda d_{1}}\right) \\
& \quad<4 \sqrt{\eta} \Gamma(1+\alpha) .
\end{aligned}
$$

Proof. (i) The Jacobian matrix for system (16) evaluated at trivial equilibrium $P_{0}=(0,0,0)$ is given by

$$
\begin{aligned}
& J(0,0,0) \\
& =\left[\begin{array}{ccc}
1+\frac{k^{\alpha}}{\Gamma(1+\alpha)} & 0 & 0 \\
0 & 1-\frac{k^{\alpha} \mu}{\Gamma(1+\alpha)} & \frac{k^{\alpha} \phi}{\Gamma(1+\alpha)} \\
0 & 0 & 1-\frac{k^{\alpha} v}{\Gamma(1+\alpha)}
\end{array}\right] .
\end{aligned}
$$

$$
\lambda_{2,3}=1-\frac{k^{\alpha}\left(\sqrt{\eta}\left(\mu+\nu+\beta b_{2}\right) \pm \sqrt{\eta\left((\mu-\nu)^{2}+4 \beta \phi+\beta b_{2}\left(2 \mu-2 \nu+\beta b_{2}\right)\right)+4 \beta \lambda d_{1}}\right)}{2 \sqrt{\eta} \Gamma(1+\alpha)} .
$$

Now it is easy to see that $\left|\lambda_{1}\right|<1$ if and only if $s^{\alpha} / \Gamma(1+\alpha)<2$ and $\left|\lambda_{2,3}\right|<1$ if and only if

$$
\begin{aligned}
& k^{\alpha}\left(\sqrt{\eta}\left(\mu+\nu+\beta b_{2}\right)\right. \\
& \left.\quad+\sqrt{\eta\left((\mu-\nu)^{2}+4 \beta \phi+\beta b_{2}\left(2 \mu-2 \nu+\beta b_{2}\right)\right)+4 \beta \lambda d_{1}}\right) \\
& \quad<4 \sqrt{\eta} \Gamma(1+\alpha) .
\end{aligned}
$$

Theorem 5. The unique positive equilibrium $\left(x^{*}, y^{*}, z^{*}\right)$ of system (16) is locally asymptotically stable if the following condition is satisfied:

$$
\begin{aligned}
\left|A_{1}+A_{3}\right| & <1+A_{2}, \\
\left|A_{1}-3 A_{3}\right| & <3-A_{2}, \\
A_{3}^{2}+A_{2}-A_{1} A_{3} & <1,
\end{aligned}
$$

where

$$
\begin{aligned}
& A_{1}=M\left(x^{*}+\mu+\nu\right)+M x^{*} \beta b_{2}-3, \\
& A_{2}=3-2 M\left(x^{*}+\mu+\nu\right)+M^{2}\left[\mu\left(y^{*} \beta+\nu\right)\right.
\end{aligned}
$$

Now it is easy to see that eigenvalues of Jacobian matrix $J(0,0,0)$ are $\lambda_{1}=1+k^{\alpha} / \Gamma(1+\alpha), \lambda_{2}=1-k^{\alpha} \mu / \Gamma(1+\alpha)$, and $\lambda_{3}=1-k^{\alpha} \nu / \Gamma(1+\alpha)$. Since $0<\alpha \leq 1$ and $s>0$ implies that $k^{\alpha} / \Gamma(1+\alpha)>0$, then it follows that $\lambda_{1}>1$. Hence $P_{0}$ is unstable.

(ii) The Jacobian matrix for system (16) evaluated at trivial equilibrium $P_{1}=(1,0,0)$ is given by

$J(1,0,0)$

$$
=\left[\begin{array}{ccc}
1-\frac{k^{\alpha}}{\Gamma(1+\alpha)} & -\frac{s^{\alpha} \beta}{\Gamma(1+\alpha)} & \frac{s^{\alpha} c \lambda}{\eta \Gamma(1+\alpha)} \\
0 & 1-\frac{s^{\alpha}\left(\mu+b_{2} \beta\right)}{\Gamma(1+\alpha)} & \frac{s^{\alpha}\left(\eta \phi+\lambda d_{1}\right)}{\eta \Gamma(1+\alpha)} \\
0 & \frac{s^{\alpha} \beta}{\Gamma(1+\alpha)} & 1-\frac{k^{\alpha} v}{\Gamma(1+\alpha)}
\end{array}\right] .
$$

The eigenvalues of Jacobian matrix $J(1,0,0)$ are given by $\lambda_{1}=$ $1-k^{\alpha} / \Gamma(1+\alpha)$ and 


$$
J\left(x^{*}, y^{*}, z^{*}\right)=\left[\begin{array}{ccc}
1-\frac{k^{\alpha} x^{*}}{\Gamma(1+\alpha)} & -\frac{k^{\alpha} x^{*} \beta}{\Gamma(1+\alpha)} & \frac{c k^{\alpha} x^{*} \eta \lambda}{\left(z^{*}+\eta\right)^{2} \Gamma(1+\alpha)} \\
\frac{k^{\alpha}\left(\mu y^{*}-\phi z^{*}\right)}{x^{*} \Gamma(1+\alpha)} & 1-\frac{k^{\alpha}\left(\mu+x^{*} \beta b_{2}\right)}{\Gamma(1+\alpha)} & \frac{k^{\alpha}\left(\left(z^{*}+\eta\right)^{2} \phi+x^{*} \eta \lambda d_{1}\right)}{\left(z^{*}+\eta\right)^{2} \Gamma(1+\alpha)} \\
\frac{k^{\alpha} y^{*} \beta}{\Gamma(1+\alpha)} & \frac{k^{\alpha} x^{*} \beta}{\Gamma(1+\alpha)} & 1-\frac{k^{\alpha} v}{\Gamma(1+\alpha)}
\end{array}\right]
$$

The characteristic polynomial of Jacobian matrix $J\left(x^{*}, y^{*}\right.$, $z^{*}$ ) evaluated at positive equilibrium is given by

$$
P(T)=T^{3}+A_{1} T^{2}+A_{2} T+A_{3},
$$

where

$$
\begin{aligned}
A_{1} & =M\left(x^{*}+\mu+\nu\right)+M x^{*} \beta b_{2}-3 \\
A_{2} & =3-2 M\left(x^{*}+\mu+\nu\right)+M^{2}\left[\mu\left(y^{*} \beta+\nu\right)\right. \\
& \left.-c L y^{*} \beta+x^{*}(\mu+\nu)-\left(x^{*}+z^{*}\right) \beta \phi\right] \\
& +M x^{*} \beta\left[\left(-2+M\left(x^{*}+\nu\right)\right) b_{2}-L M d_{1}\right], \\
A_{3} & =M\left[\nu+x^{*}(M \mu-1)(M \nu-1)-M^{2}\left(x^{*}\right)^{2} \beta \phi\right. \\
& \left.+M x^{*} \beta\left(1+M y^{*} \beta\right) \phi\right]+c L M{ }^{2} \beta\left(y^{*}-2 M y^{*} \mu\right. \\
& \left.+M z^{*} \phi\right)+M(M \nu-1)\left(M y^{*} \beta \mu-M z^{*} \beta \phi-\mu\right) \\
& +M x^{*} \beta\left[\left(1-M\left(x^{*}+c L M y^{*} \beta\right)\right.\right. \\
& \left.+M\left(M x^{*}-1\right) \nu\right) b_{2}+L M\left(1-M x^{*}\right. \\
& \left.\left.+M y^{*} \beta\right) d_{1}\right]-1, \\
M & =\frac{s^{\alpha}}{\Gamma(1+\alpha)} . \\
& \left.+z^{*}+\eta\right)^{2},
\end{aligned}
$$

Now applying the Jury condition [11], the unique positive equilibrium point $\left(x^{*}, y^{*}, z^{*}\right)$ is locally asymptotically stable if the following conditions are satisfied:

$$
\begin{aligned}
\left|A_{1}+A_{3}\right| & <1+A_{2}, \\
\left|A_{1}+3 A_{3}\right| & <3-A_{2}, \\
A_{3}^{2}+A_{2}-A_{1} A_{3} & <1 .
\end{aligned}
$$

In order to study the Neimark-Sacker bifurcation in system (16), we need the following explicit criterion of Hopf bifurcation.
Lemma 6 (see [24]). Consider an n-dimensional discrete dynamical system $X_{k+1}=f_{\mu}\left(X_{k}\right)$, where $\mu \in \mathbb{R}$ is bifurcation parameter. Let $X^{*}$ be a fixed point of $f_{\mu}$ and the characteristic polynomial for Jacobian matrix $J\left(X^{*}\right)=\left(a_{i j}\right)_{n \times n}$ of $n$-dimensional map $f_{\mu}$ is given by

$$
P_{\mu}(\lambda)=\lambda^{n}+a_{1} \lambda^{n-1}+\cdots+a_{n-1} \lambda+a_{n},
$$

where $a_{i}=a_{i}(\mu, u), i=1,2, \ldots, n$ and $u$ is control parameter or another parameter to be determined. Let $\Delta_{0}^{ \pm}(\mu, u)=1$, $\Delta_{1}^{ \pm}(\mu, u), \ldots, \Delta_{n}^{ \pm}(\mu, u)$ be a sequence of determinants defined by $\Delta_{i}^{ \pm}(\mu, u)=\operatorname{det}\left(M_{1} \pm M_{2}\right), i=1,2, \ldots, n$, where

$$
\begin{aligned}
M_{1} & =\left[\begin{array}{ccccc}
1 & a_{1} & a_{2} & \cdots & a_{i-1} \\
0 & 1 & a_{1} & \cdots & a_{i-2} \\
0 & 0 & 1 & \cdots & a_{i-3} \\
\cdots & \cdots & \cdots & \cdots & \cdots \\
0 & 0 & 0 & \cdots & 1
\end{array}\right], \\
M_{2} & =\left[\begin{array}{ccccc}
a_{n-i+1} & a_{n-i+2} & \cdots & a_{n-1} & a_{n} \\
a_{n-i+2} & a_{n-i+3} & \cdots & a_{n} & 0 \\
\cdots & \cdots & \cdots & \cdots & \cdots \\
a_{n-1} & a_{n} & \cdots & 0 & 0 \\
a_{n} & 0 & \cdots & 0 & 0
\end{array}\right] .
\end{aligned}
$$

Moreover, the following conditions hold:,

(H1) Eigenvalue assignment: $\Delta_{n-1}^{-}\left(\mu_{0}, u\right)=0, \Delta_{n-1}^{+}\left(\mu_{0}, u\right)>$ $0, P_{\mu_{0}}(1)>0,(-1)^{n} P_{\mu_{0}}(-1)>0, \Delta_{i}^{ \pm}\left(\mu_{0}, u\right)>0, i=n-$ $3, n-5, \ldots, 1$ (or 2$)$, when $n$ is even or odd, respectively.

(H2) Transversality condition: $\left((d / d \mu)\left(\Delta_{n-1}^{-}(\mu, u)\right)\right)_{\mu=\mu_{0}} \neq$ 0 .

(H3) Nonresonance condition: $\cos (2 \pi / m) \neq \psi$, or resonance condition $\cos (2 \pi / m)=\psi$, where $m=3,4,5, \ldots$, and $\psi=-1+0.5 P_{\mu_{0}}(1) \Delta_{n-3}^{-}\left(\mu_{0}, u\right) / \Delta_{n-2}^{+}\left(\mu_{0}, u\right)$. Then Neimark-Sacker bifurcation occurs at $\mu_{0}$.

The following result shows that system (16) undergoes Neimark-Sacker bifurcation if we take $\mu$ as bifurcation parameter. 
Theorem 7. The unique positive equilibrium point of system (16) undergoes Neimark-Sacker bifurcation if the following conditions hold:

$$
\begin{aligned}
1-A_{2}+A_{3}\left(A_{1}-A_{3}\right) & =0, \\
1+A_{2}-A_{3}\left(A_{1}+A_{3}\right) & >0, \\
1+A_{1}+A_{2}+A_{3} & >0 \\
1-A_{1}+A_{2}-A_{3} & >0,
\end{aligned}
$$

where $A_{1}, A_{2}$, and $A_{3}$ are given in (32).

Proof. According to Lemma 6, for $n=3$, we have in (32) the characteristic polynomial of system (16) evaluated at its unique positive equilibrium, then we obtain the following equalities and inequalities:

$$
\begin{aligned}
\Delta_{2}^{-}(\mu) & =1-A_{2}+A_{3}\left(A_{1}-A_{3}\right)=0, \\
\Delta_{2}^{+}(\mu) & =1+A_{2}-A_{3}\left(A_{1}+A_{3}\right)>0, \\
P_{\mu}(1) & =1+A_{1}+A_{2}+A_{3}>0, \\
(-1)^{3} P_{\mu}(-1) & =1-A_{1}+A_{2}-A_{3}>0 .
\end{aligned}
$$

\section{Chaos Control}

Controlling chaos in discrete-time models is a topic of great interest for many researchers in recent times, and practical methods can be used in many fields such as biochemistry, cardiology, communications, physics laboratories, and turbulence [25]. Chaos control in discrete-time models can be obtained by using various methods. In this section, we concentrate on two procedures only, that is, pole-placement technique which is based on feedback control methodology and the other one is hybrid control based on feedback control strategy and parameter perturbation.

First, we study chaos controlling technique based on poleplacement methodology introduced by Romeiras et al. [26] (also see [27]), which may be treated as generalized OGY method studied for the first time by Ott et al. [28]. In order to apply pole-placement technique to system (16), we rewrite system (16) as follows:

$$
\begin{aligned}
x_{n+1} & \\
= & x_{n} \\
& +\frac{k^{\alpha}}{\Gamma(\alpha+1)}\left[x_{n}\left(1-x_{n}\right)+\frac{\lambda c x_{n} z_{n}}{\eta+z_{n}}-\beta x_{n} y_{n}\right] \\
= & f\left(x_{n}, y_{n}, z_{n}, \beta\right), \\
y_{n+1} & \\
= & y_{n} \\
& +\frac{k^{\alpha}}{\Gamma(\alpha+1)}\left[\frac{\lambda d_{1} x_{n} z_{n}}{\eta+z_{n}}+\phi z_{n}-b_{2} \beta x_{n} y_{n}-\mu y_{n}\right] \\
= & g\left(x_{n}, y_{n}, z_{n}, \beta\right), \\
z_{n+1}= & z_{n}+\frac{k^{\alpha}}{\Gamma(\alpha+1)}\left[\beta x_{n} y_{n}-v z_{n}\right] \\
= & h\left(x_{n}, y_{n}, z_{n}, \beta\right),
\end{aligned}
$$

where $\beta$ is taken as control parameter. Moreover, $\beta$ is restricted to lie in some small interval $\left|\beta-\beta_{0}\right|<\delta$ with $\delta>0$ and $\beta_{0}$ denotes the nominal value belonging to chaotic region. We apply the stabilizing feedback control strategy in order to move the trajectory towards the desired orbit. Assume that $\left(x^{*}, y^{*}, z^{*}\right)$ is unstable equilibrium point of system (16) in chaotic region produced by Neimark-Sacker bifurcation; then system (39) can be approximated in the neighborhood of the unstable equilibrium point $\left(x^{*}, y^{*}, z^{*}\right)$ by the following linear map:

$$
\left[\begin{array}{c}
x_{n+1}-x^{*} \\
y_{n+1}-y^{*} \\
z_{n+1}-z^{*}
\end{array}\right] \approx A\left[\begin{array}{c}
x_{n}-x^{*} \\
y_{n}-y^{*} \\
z_{n}-z^{*}
\end{array}\right]+B\left[\beta-\beta_{0}\right],
$$

where

$$
\begin{aligned}
& A=\left[\begin{array}{lll}
\frac{\partial f\left(x^{*}, y^{*}, z^{*}, \beta_{0}\right)}{\partial x} & \frac{\partial f\left(x^{*}, y^{*}, z^{*}, \beta_{0}\right)}{\partial y} & \frac{\partial f\left(x^{*}, y^{*}, z^{*}, \beta_{0}\right)}{\partial z} \\
\frac{\partial g\left(x^{*}, y^{*}, z^{*}, \beta_{0}\right)}{\partial x} & \frac{\partial g\left(x^{*}, y^{*}, z^{*}, \beta_{0}\right)}{\partial y} & \frac{\partial g\left(x^{*}, y^{*}, z^{*}, \beta_{0}\right)}{\partial y} \\
\frac{\partial h\left(x^{*}, y^{*}, z^{*}, \beta_{0}\right)}{\partial x} & \frac{\partial h\left(x^{*}, y^{*}, z^{*}, \beta_{0}\right)}{\partial y} & \frac{\partial h\left(x^{*}, y^{*}, z^{*}, \beta_{0}\right)}{\partial y}
\end{array}\right] \\
& B=\left[\begin{array}{c}
\frac{\partial f\left(x^{*}, y^{*}, z^{*}, \beta_{0}\right)}{\partial \beta} \\
\frac{\partial g\left(x^{*}, y^{*}, z^{*}, \beta_{0}\right)}{\partial \beta}
\end{array}\right]=\left[\begin{array}{c}
-\frac{k^{\alpha} x^{*} y^{*}}{\Gamma(\alpha+1)} \\
-\frac{k^{\alpha} b_{2} x^{*} y^{*}}{\Gamma(\alpha+1)} \\
\frac{k^{\alpha} x^{*} y^{*}}{\Gamma(\alpha+1)}
\end{array}\right]
\end{aligned}
$$


It is easy to see that system (39) is controllable provided that the following matrix

$$
C=\left[B: A B: A^{2} B\right]
$$

has rank 3. Next, we assume that $\left[\beta-\beta_{0}\right]=-K\left[\begin{array}{c}x_{n}-x^{*} \\ y_{n}-y^{*} \\ z_{n}-z^{*}\end{array}\right]$, where $K=\left[\begin{array}{lll}k_{1} & k_{2} & k_{3}\end{array}\right]$; then system (40) can be written as

$$
\left[\begin{array}{c}
x_{n+1}-x^{*} \\
y_{n+1}-y^{*} \\
z_{n+1}-z^{*}
\end{array}\right] \approx[A-B K]\left[\begin{array}{c}
x_{n}-x^{*} \\
y_{n}-y^{*} \\
z_{n}-z^{*}
\end{array}\right]
$$

Moreover, equilibrium point $\left(x^{*}, y^{*}, z^{*}\right)$ is locally asymptotically stable if and only if all three eigenvalues of the matrix $A-B K$, say, $\mu_{1}, \mu_{2}$, and $\mu_{3}$, lie in an open unit disk. These eigenvalues are known as regulator poles, and problem of placing these regulator poles at suitable position is known as pole-placement problem. Assume that rank of the matrix $C$ is 3 ; therefore pole-placement problem has a unique solution. Next, we assume that $\lambda^{3}+\alpha_{1} \lambda^{2}+\alpha_{2} \lambda+\alpha_{3}=0$ is characteristic equation of $A$ and $\mu^{3}+\tau_{1} \mu^{2}+\tau_{2} \mu+\tau_{3}=0$ is characteristic equation of $A-B K$; then unique solution of the pole-placement problem is given by

$$
K=\left[\begin{array}{lll}
\tau_{3}-\alpha_{3} & \tau_{2}-\alpha_{2} & \tau_{1}-\alpha_{1}
\end{array}\right] T^{-1},
$$

where $T=C M$ and

$$
M=\left[\begin{array}{ccc}
\alpha_{2} & \alpha_{1} & 1 \\
\alpha_{1} & 1 & 0 \\
1 & 0 & 0
\end{array}\right]
$$

Next in order to control Neimark-Sacker bifurcation in system (16), we apply hybrid control feedback methodology $[29,30]$. Assuming that system (16) undergoes NeimarkSacker bifurcation at equilibrium point $\left(x^{*}, y^{*}, z^{*}\right)$, then corresponding controlled system can be written as

$$
\begin{aligned}
x_{n+1} & \rho x_{n} \\
& +\frac{\rho k^{\alpha}}{\Gamma(\alpha+1)}\left[x_{n}\left(1-x_{n}\right)+\frac{\lambda c x_{n} z_{n}}{\eta+z_{n}}-\beta x_{n} y_{n}\right] \\
& +(1-\rho) x_{n}, \\
y_{n+1} & \rho y_{n} \\
& +\frac{\rho k^{\alpha}}{\Gamma(\alpha+1)}\left[\frac{\lambda d_{1} x_{n} z_{n}}{\eta+z_{n}}+\phi z_{n}-b_{2} \beta x_{n} y_{n}-\mu y_{n}\right] \\
& +(1-\rho) y_{n}, \\
z_{n+1}= & \rho z_{n}+\frac{\rho k^{\alpha}}{\Gamma(\alpha+1)}\left[\beta x_{n} y_{n}-\nu z_{n}\right]+(1-\rho) z_{n},
\end{aligned}
$$

where $0<\rho<1$ and controlled strategy in (45) is a combination of both parameter perturbation and feedback control. Moreover, by suitable choice of controlled parameter $\alpha$, the Neimark-Sacker bifurcation of the equilibrium point $\left(x^{*}, y^{*}, z^{*}\right)$ of controlled system (45) can be advanced (delayed) or even completely eliminated. The Jacobian matrix of controlled system (45) evaluated at positive equilibrium $\left(x^{*}, y^{*}, z^{*}\right)$ is given by

$$
\left[\begin{array}{ccc}
1-\frac{\rho k^{\alpha} x^{*}}{\Gamma(1+\alpha)} & -\frac{\rho k^{\alpha} x^{*} \beta}{\Gamma(1+\alpha)} & \frac{\rho c k^{\alpha} x^{*} \eta \lambda}{\left(z^{*}+\eta\right)^{2} \Gamma(1+\alpha)} \\
\frac{\rho k^{\alpha}\left(\mu y^{*}-\phi z^{*}\right)}{x^{*} \Gamma(1+\alpha)} & 1-\frac{\rho k^{\alpha}\left(\mu+x^{*} \beta b_{2}\right)}{\Gamma(1+\alpha)} & \frac{\rho k^{\alpha}\left(\left(z^{*}+\eta\right)^{2} \phi+x^{*} \eta \lambda d_{1}\right)}{\left(z^{*}+\eta\right)^{2} \Gamma(1+\alpha)} \\
\frac{\rho k^{\alpha} y^{*} \beta}{\Gamma(1+\alpha)} & \frac{\rho k^{\alpha} x^{*} \beta}{\Gamma(1+\alpha)} & 1-\frac{\rho k^{\alpha} \nu}{\Gamma(1+\alpha)}
\end{array}\right] .
$$

Then, positive equilibrium $\left(x^{*}, y^{*}, z^{*}\right)$ of the controlled system (45) is locally asymptotically stable if roots of the characteristic polynomial of (47) lie in an open unit disk.

\section{Numerical Simulations}

Example 8. First, we take $\alpha=0.38, k=0.005, c=0.8$, $\lambda=2.7, \eta=1.1, d_{1}=0.7, b_{2}=1.4, \phi=2.7, \mu=4, \nu=1.5$, $x_{0}=0.48, y_{0}=0.1, z_{0}=0.32$, and $\beta \in[8,14]$ in system (16); then system (16) undergoes Neimark-Sacker bifurcation when $\beta$ is taken as bifurcation parameter. In this case, at $\beta=$ 10.148 , the unique positive equilibrium point of system (16) is $\left(x^{*}, y^{*}, z^{*}\right)=(0.4784797606357651,0.0996595753165892$,
$0.32260552713822044)$. The characteristic polynomial equation evaluated at this positive equilibrium is given by

$$
\begin{aligned}
P(T)= & T^{3}-1.0798313086203364 T^{2} \\
& -0.8127303335375109 T \\
& +0.9108087500517679=0 .
\end{aligned}
$$

The roots of (48) are $T_{1}=-0.9107182237825987$ and $T_{2,3}=$ $0.9952747662014675 \pm 0.097609122147403 i$ with $\left|T_{2,3}\right|=1$. Moreover, we have

$$
\begin{aligned}
\Delta_{2}^{-}(\beta) & =1-A_{2}+A_{3}\left(A_{1}-A_{3}\right)=0 \\
\Delta_{2}^{+}(\beta) & =1+A_{2}-A_{3}\left(A_{1}+A_{3}\right)=0.341217 \\
& >0
\end{aligned}
$$




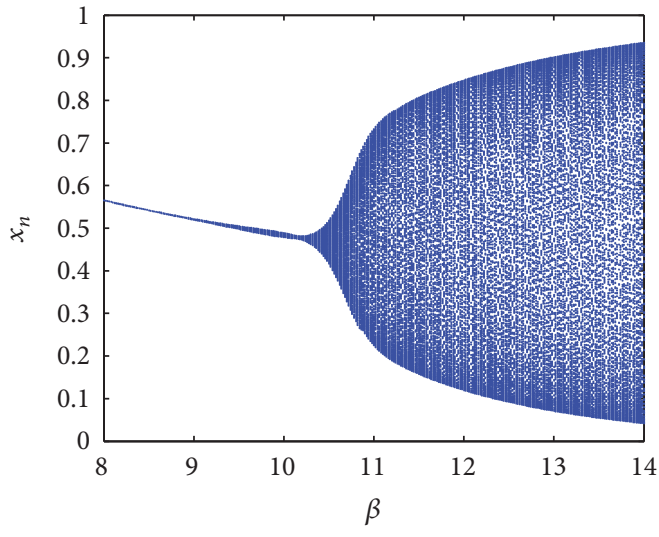

(a) Bifurcation diagram for $x_{n}$

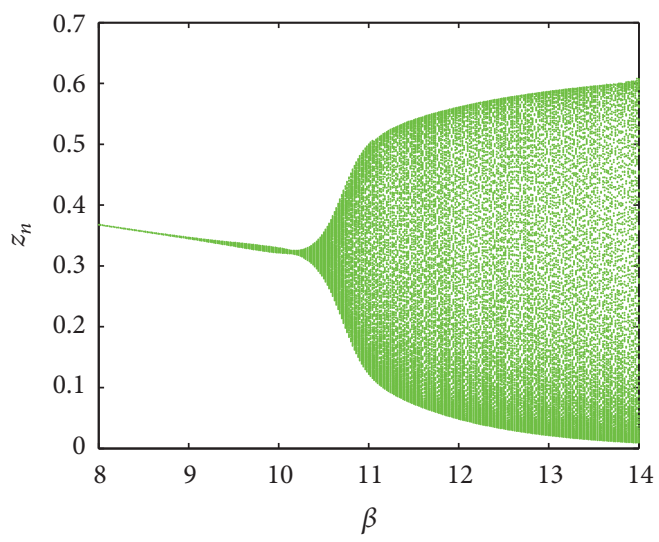

(c) Bifurcation diagram for $z_{n}$

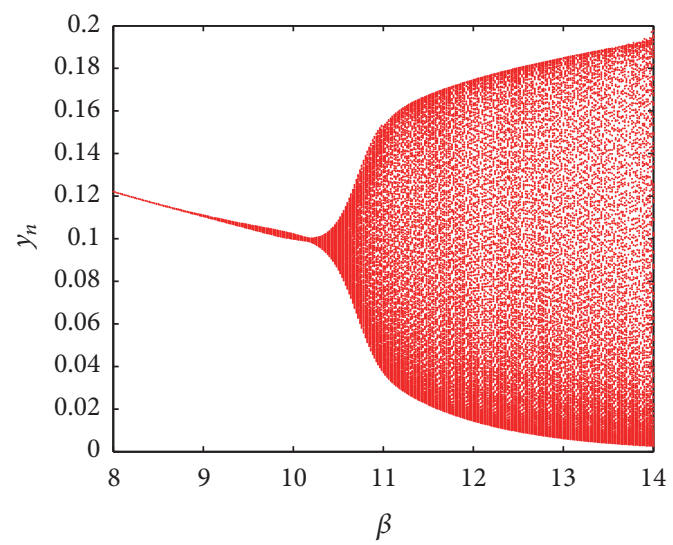

(b) Bifurcation diagram for $y_{n}$

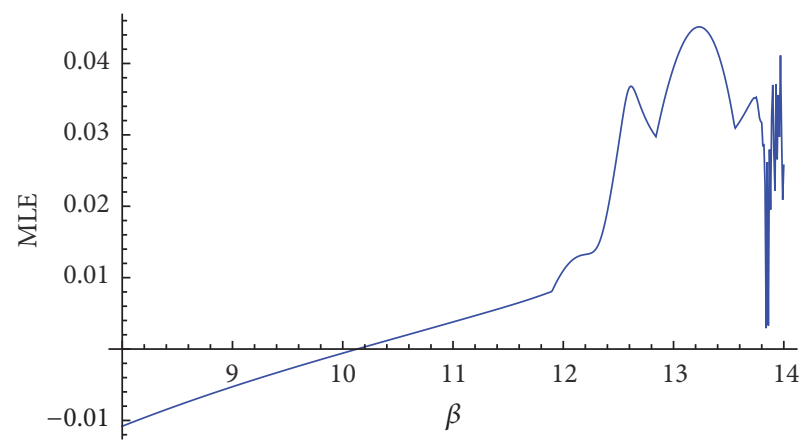

(d) Maximum Lyapunov exponents

FIGURE 1: Bifurcation diagrams and MLE for system (16) with $\alpha=0.38, k=0.005, c=0.8, \lambda=2.7, \eta=1.1, d_{1}=0.7, b_{2}=1.4, \phi=2.7, \mu=4$, $\nu=1.5, x_{0}=0.48, y_{0}=0.1, z_{0}=0.32$, and $\beta \in[8,14]$.

$$
\begin{array}{r}
P_{\beta}(1)=1+A_{1}+A_{2}+A_{3}=0.0182471>0, \\
(-1)^{3} P_{\beta}(-1)=1-A_{1}+A_{2}-A_{3}=0.356292>0 .
\end{array}
$$

Hence, according to Theorem 7 , the conditions of Neimark-Sacker bifurcation are obtained near the positive equilibrium point $\left(x^{*}, y^{*}, z^{*}\right)=(0.4784797606357651$, $0.0996595753165892,0.32260552713822044)$ at the critical value of Neimark-Sacker bifurcation $\beta=10.148$.

Furthermore, Figure 1 shows that all three populations undergo Neimark-Sacker bifurcation (see Figures 1(a), 1(b), and 1(c)) and corresponding maximum Lyapunov exponents (MLEs) are shown in Figure 1(d). These MLEs confirm the existence of the chaotic sets. In general, the positive Lyapunov exponent is considered to be one of the characteristics implying the existence of chaos.

Example 9. Next we take $\alpha=0.3, k=0.03, c=0.3, \lambda=2.1$, $\eta=1.1, d_{1}=1.7, b_{2}=1.4, \phi=2.5, \mu=1.5, \nu=1.3$, $x_{0}=0.37, y_{0}=0.23, z_{0}=0.2$, and $\beta \in[2,5]$ in system (16); then system (16) undergoes Neimark-Sacker bifurcation when $\beta$ is taken as bifurcation parameter. In this case, at $\beta=3.13$, the unique positive equilibrium point of system (16) is $\left(x^{*}, y^{*}, z^{*}\right)=(0.3691168623529498,0.2335056090113552$, $0.20752106517420343)$. The characteristic polynomial equation evaluated at this positive equilibrium is given by

$$
\begin{aligned}
P(T)= & T^{3}-1.137303699628256 T^{2} \\
& -0.5469293363877263 T \\
& +0.7989950157191528=0 .
\end{aligned}
$$

The roots of (48) are $T_{1}=-0.798954$ and $T_{2,3}=$ $0.9681288296244075 \pm 0.2505552964214181 i$ with $\left|T_{2,3}\right|=1$. Moreover, we verify the conditions of Theorem 7 as follows:

$$
\begin{aligned}
\Delta_{2}^{-}(\beta) & =1-A_{2}+A_{3}\left(A_{1}-A_{3}\right)=0, \\
\Delta_{2}^{+}(\beta) & =1+A_{2}-A_{3}\left(A_{1}+A_{3}\right)=0.723378 \\
& >0 \\
P_{\beta}(1) & =1+A_{1}+A_{2}+A_{3}=0.114762>0, \\
(-1)^{3} P_{\beta}(-1) & =1-A_{1}+A_{2}-A_{3}=0.791379>0 .
\end{aligned}
$$

Hence, according to Theorem 7, the conditions of Neimark-Sacker bifurcation are obtained near the positive 


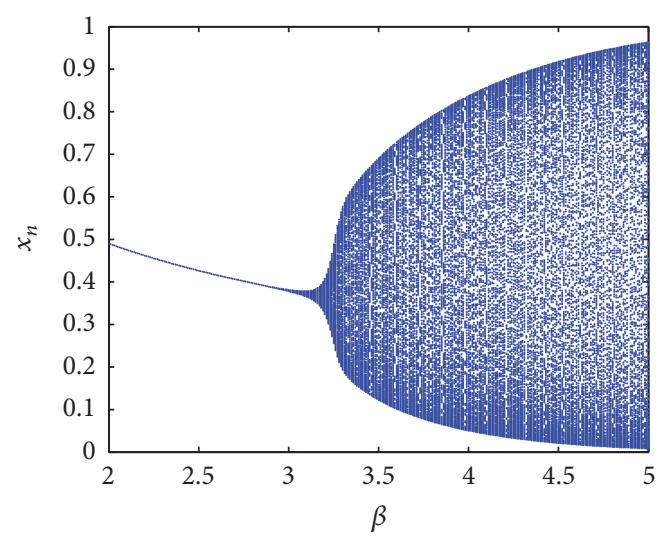

(a) Bifurcation diagram for $x_{n}$

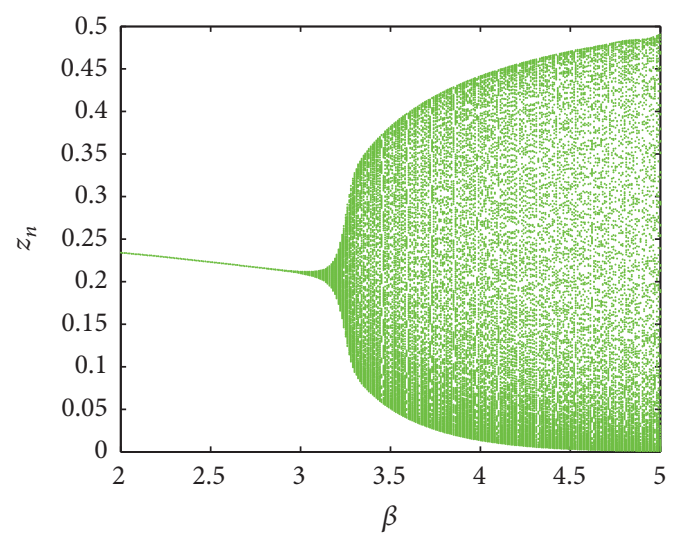

(c) Bifurcation diagram for $z_{n}$

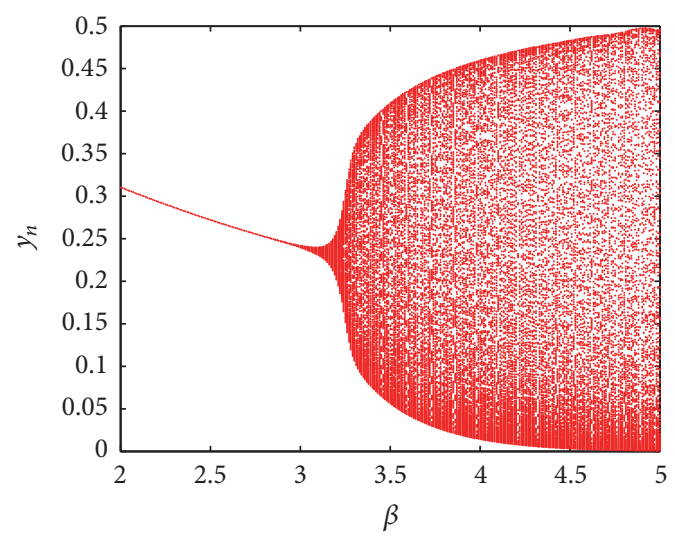

(b) Bifurcation diagram for $y_{n}$

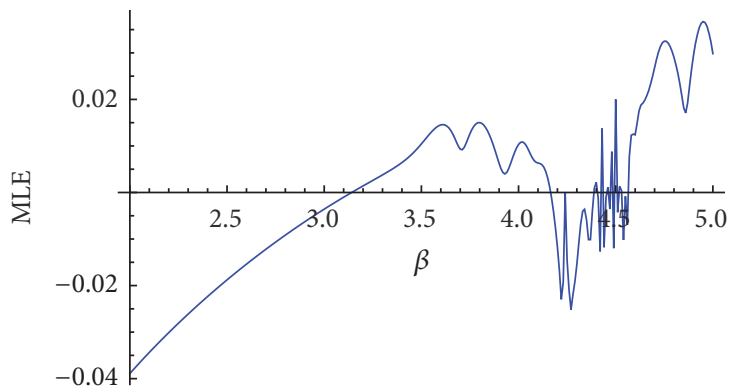

(d) Maximum Lyapunov exponents

Figure 2: Bifurcation diagrams and MLE for system (16) with $\alpha=0.3, k=0.03, c=0.3, \lambda=2.1, \eta=1.1, d_{1}=1.7, b_{2}=1.4, \phi=2.5, \mu=1.5$, $\nu=1.3, x_{0}=0.37, y_{0}=0.23, z_{0}=0.2$, and $\beta \in[2,5]$.

equilibrium point $\left(x^{*}, y^{*}, z^{*}\right)=(0.3691168623529498$, $0.2335056090113552,0.20752106517420343)$ at the critical value of Neimark-Sacker bifurcation $\beta=3.13$.

Furthermore, Figure 2 shows that all three populations undergo Neimark-Sacker bifurcation (see Figures 2(a), 2(b), and 2(c)) and corresponding maximum Lyapunov exponents (MLEs) are shown in Figure 2(d).

Next, we fix the value of $\beta=2.98$. In Figure 3, the plot of $x_{n}$ is shown in Figure 3(a), the plot of $y_{n}$ is shown in Figure 3(b), the plot of $z_{n}$ is shown in Figure 3(c), and phase portrait of system (16) is shown in Figure 3(d). It is clear from Figure 3 that system (16) has unique positive equilibrium point which is locally asymptotically stable.

Furthermore, the phase portraits of system (16) for different values of $\beta$ are shown in Figure 4 .

Example 10. We choose the parametric values $\alpha=0.3, k=$ $0.03, c=0.3, \lambda=2.1, \eta=1.1, d_{1}=1.7, b_{2}=1.4, \phi=2.5$, $\mu=1.5, v=1.3$, and $\beta=3.2$ and initial conditions $x_{0}=$ $0.37, y_{0}=0.23$, and $z_{0}=0.2$ in system (16); then system (16) has a unique positive equilibrium point $\left(x^{*}, y^{*}, z^{*}\right)$ $=(0.363861,0.229828,0.205847)$ which is unstable and Figure 4(c) shows a chaotic attractor for system (16) for these parametric values. So, one can take $\beta_{0}=3.2$ in order to apply pole-placement technique for system (16). In this case, the corresponding controlled system is given by

$$
\begin{aligned}
& x_{n+1}=x_{n}+\frac{k^{\alpha}}{\Gamma(\alpha+1)}\left[x_{n}\left(1-x_{n}\right)+\frac{\lambda c x_{n} z_{n}}{\eta+z_{n}}-(3.2\right. \\
& \left.-k_{1}\left(x_{n}-x^{*}\right)-k_{2}\left(y_{n}-y^{*}\right)-k_{3}\left(z_{n}-z^{*}\right)\right) \\
& \left.\quad x_{n} y_{n}\right], \\
& y_{n+1}=y_{n}+\frac{k^{\alpha}}{\Gamma(\alpha+1)}\left[\frac{\lambda d_{1} x_{n} z_{n}}{\eta+z_{n}}+\phi z_{n}-b_{2}(3.2\right. \\
& \left.-k_{1}\left(x_{n}-x^{*}\right)-k_{2}\left(y_{n}-y^{*}\right)-k_{3}\left(z_{n}-z^{*}\right)\right) x_{n} y_{n} \\
& \left.-\mu y_{n}\right], \\
& z_{n+1}=z_{n}+\frac{k^{\alpha}}{\Gamma(\alpha+1)}\left[\left(3.2-k_{1}\left(x_{n}-x^{*}\right)\right.\right. \\
& \left.\left.-k_{2}\left(y_{n}-y^{*}\right)-k_{3}\left(z_{n}-z^{*}\right)\right) x_{n} y_{n}-v z_{n}\right],
\end{aligned}
$$

where $\alpha=0.3, k=0.03, c=0.3, \lambda=2.1, \eta=1.1, d_{1}=1.7$, $b_{2}=1.4, \phi=2.5, \mu=1.5, v=1.3, K=\left[\begin{array}{lll}k_{1} & k_{2} & k_{3}\end{array}\right]$, and 


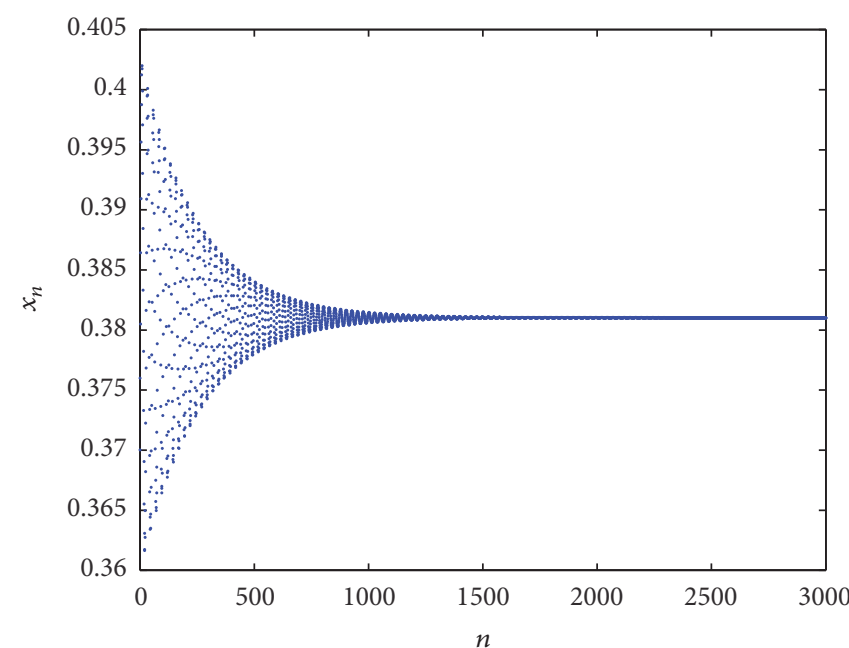

(a) Plot of $x_{n}$ for system (16)

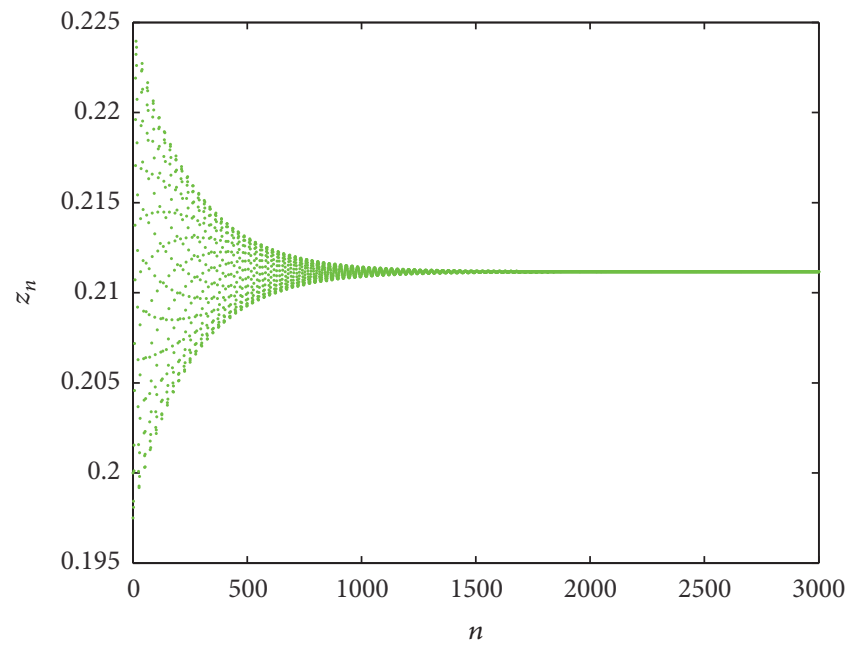

(c) Plot of $z_{n}$ for system (16)

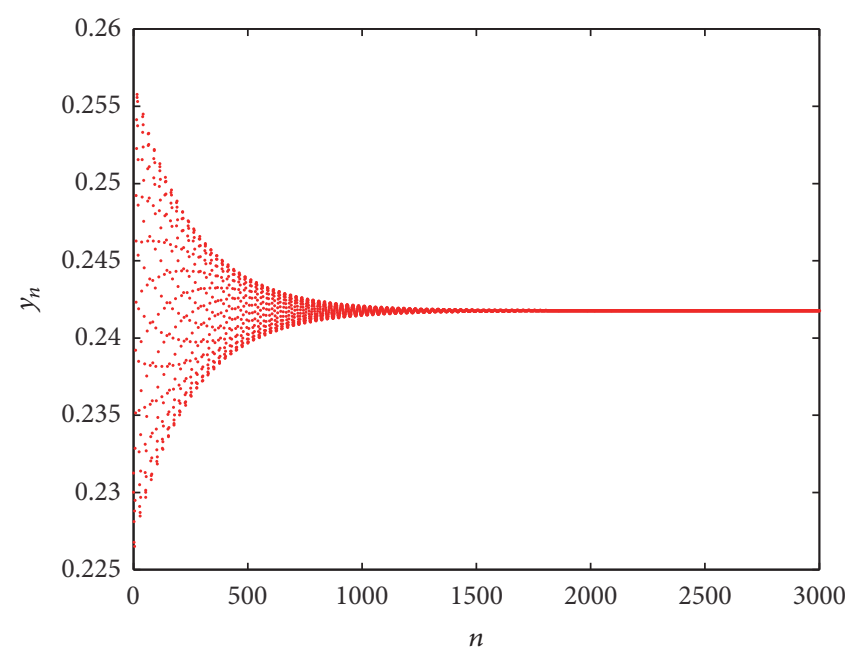

(b) Plot of $y_{n}$ for system (16)

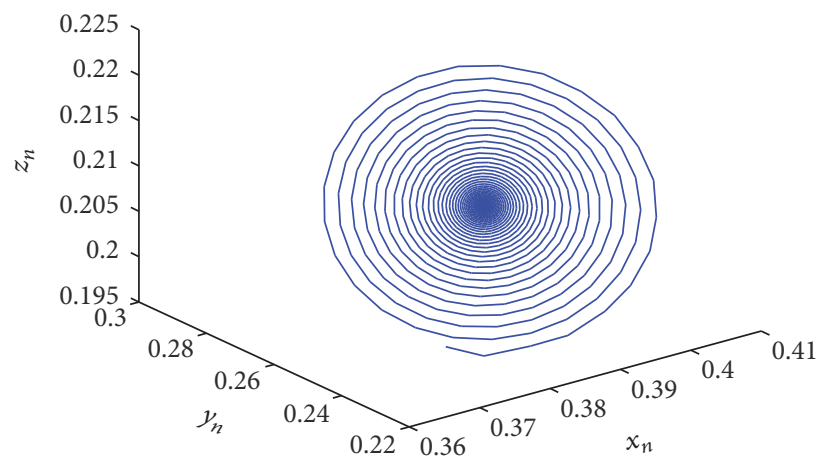

(d) Phase portrait of system (16)

FIGURE 3: Plots for system (16) with $\alpha=0.3, k=0.03, c=0.3, \lambda=2.1, \eta=1.1, d_{1}=1.7, b_{2}=1.4, \phi=2.5, \mu=1.5, v=1.3, x_{0}=0.37$, $y_{0}=0.23, z_{0}=0.2$, and $\beta=2.98$.

$\left(x^{*}, y^{*}, z^{*}\right)=(0.363861,0.229828,0.205847)$. Furthermore, we have

$$
\begin{aligned}
& A=\left[\begin{array}{ccc}
0.8584036593529867 & -0.45310829007044245 & 0.05754406574949606 \\
-0.18168256820150672 & -0.21807534869130007 & 1.2989559435682787 \\
0.28619923857656004 & 0.45310829007044245 & 0.49410608975301007
\end{array}\right], \\
& B=\left[\begin{array}{c}
-0.03254276321257405 \\
-0.04555986849760366 \\
0.03254276321257405
\end{array}\right], \\
& C=\left[\begin{array}{ccc}
-0.03254276321257405 & -0.005418630010373265 & -0.03178440171863723 \\
-0.04555986849760366 & 0.05811955270088685 & -0.029716479899874457 \\
0.03254276321257405 & -0.01387769068267633 & 0.01792659188284191
\end{array}\right] .
\end{aligned}
$$




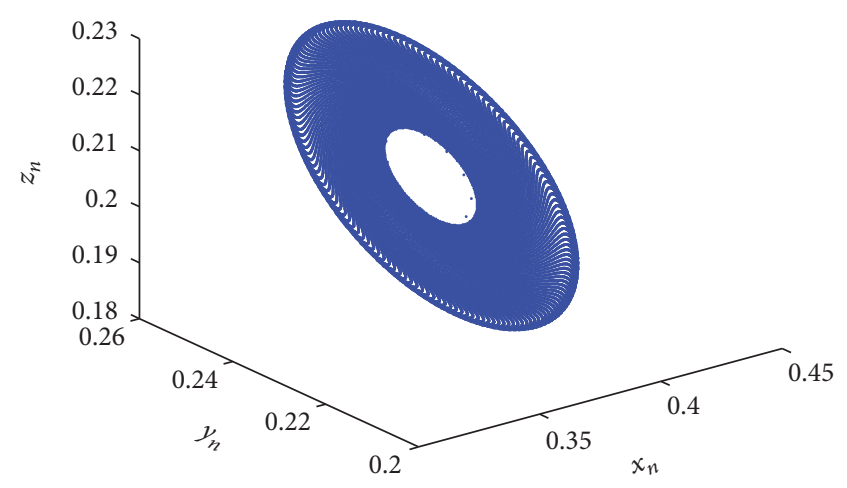

(a) Phase portrait of system (16) for $\beta=3.133$

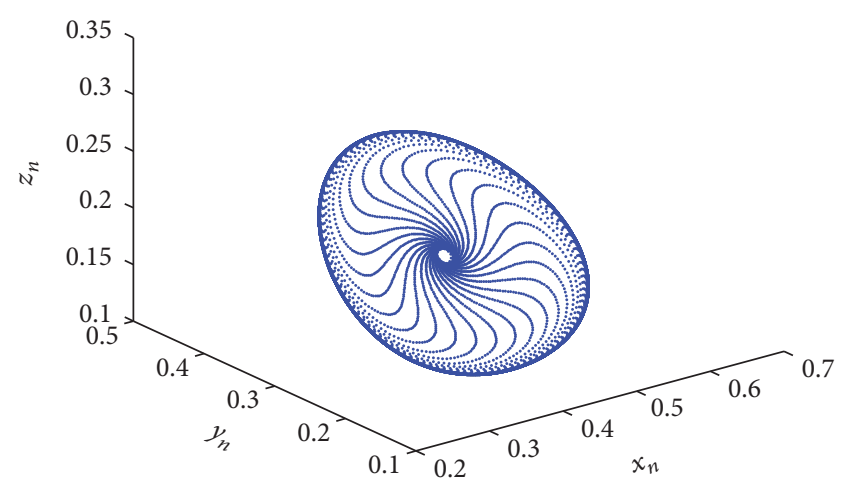

(c) Phase portrait of system (16) for $\beta=3.2$

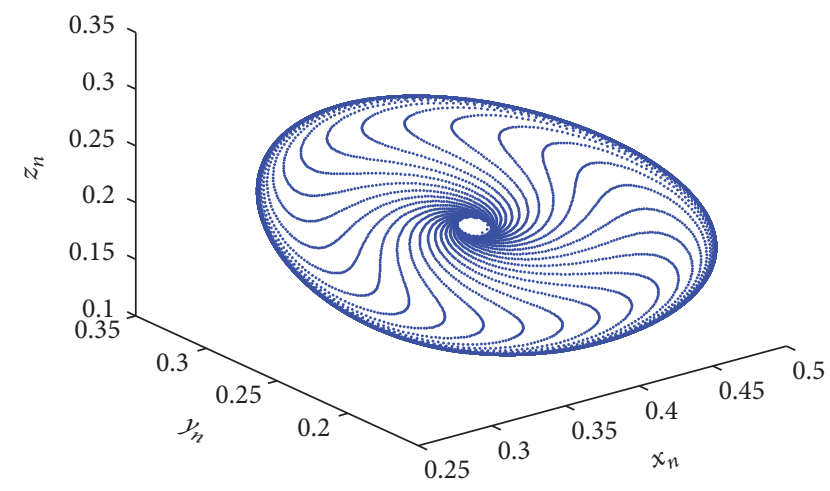

(b) Phase portrait of system (16) for $\beta=3.18$

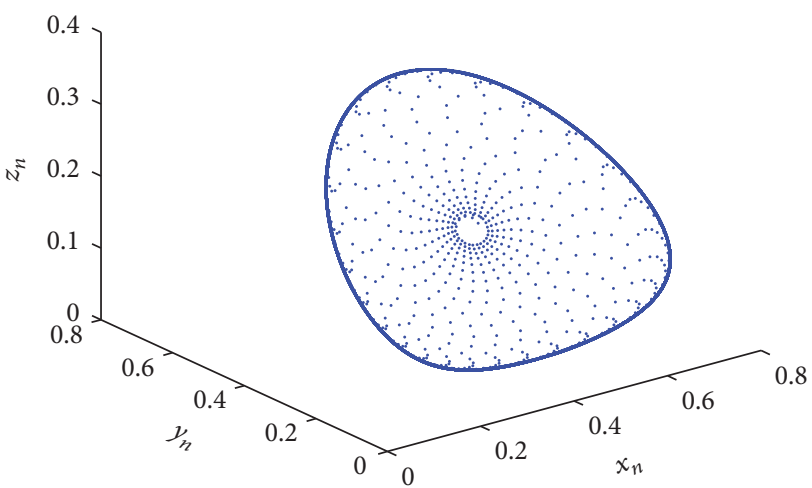

(d) Phase portrait of system (16) for $\beta=3.5$

FIGURE 4: Phase portraits of system (16) with different values of $\beta$ and taking $\alpha=0.3, k=0.03, c=0.3, \lambda=2.1, \eta=1.1, d_{1}=1.7, b_{2}=1.4$, $\phi=2.5, \mu=1.5, v=1.3, x_{0}=0.37, y_{0}=0.23$, and $z_{0}=0.2$.

Then it is easy to check that matrix $C$ is of rank 3. Thus system (52) is controllable and its Jacobian matrix at $\left(x^{*}, y^{*}, z^{*}\right)=(0.363861,0.229828,0.205847)$ is given by

$$
A-B K=\left[\begin{array}{ccc}
0.858404+0.0325428 k_{1} & -0.453108+0.0325428 k_{2} & 0.0575441+0.0325428 k_{3} \\
-0.181683+0.0455599 k_{1} & -0.218075+0.0455599 k_{2} & 1.29896+0.0455599 k_{3} \\
0.286199-0.0325428 k_{1} & 0.453108-0.0325428 k_{2} & 0.494106-0.0325428 k_{3}
\end{array}\right] .
$$

The characteristic equation of $A-B K$ is given by

$$
\begin{aligned}
& P(T)=T^{3}-\left(1.13443+0.0325428 k_{1}+0.0455599 k_{2}\right. \\
& \left.\quad-0.0325428 k_{3}\right) T^{2}-\left(0.558165-0.031499 k_{1}\right. \\
& \left.\quad-0.109804 k_{2}+0.0507953 k_{3}\right) T+0.807993 \\
& \quad-0.00747308 k_{1}-0.0702194 k_{2}+0.0155057 k_{3} \\
& \quad=0 .
\end{aligned}
$$

Then a necessary and sufficient condition for all roots of the equation (55) to lie inside the unit disk is

$$
\begin{aligned}
\left|A_{2}+A_{0}\right| & <1+A_{1}, \\
\left|A_{2}-3 A_{0}\right| & <3-A_{1}, \\
A_{0}^{2}+A_{1}-A_{0} A_{2} & <1,
\end{aligned}
$$

where $A_{2}=-1.13443-0.0325428 k_{1}-0.0455599 k_{2}+$ $0.0325428 k_{3}, A_{1}=-0.558165+0.031499 k_{1}+0.109804 k_{2}-$ $0.0507953 k_{3}$, and $A_{0}=-0.807993+0.00747308 k_{1}+$ $0.0702194 k_{2}-0.0155057 k_{3}$. For simplicity, if we choose $k_{1}=$ 


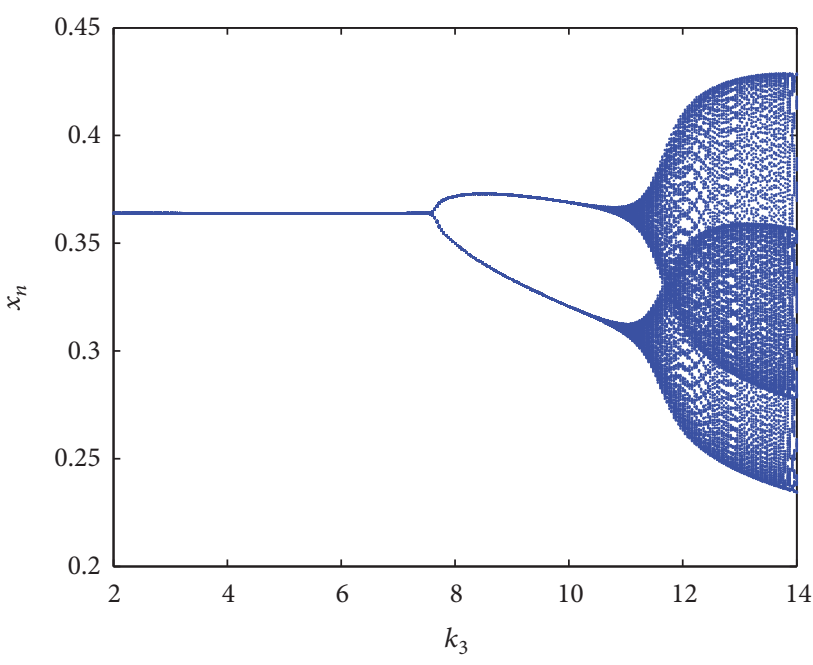

(a) Bifurcation diagram of $x_{n}$ for system (52)

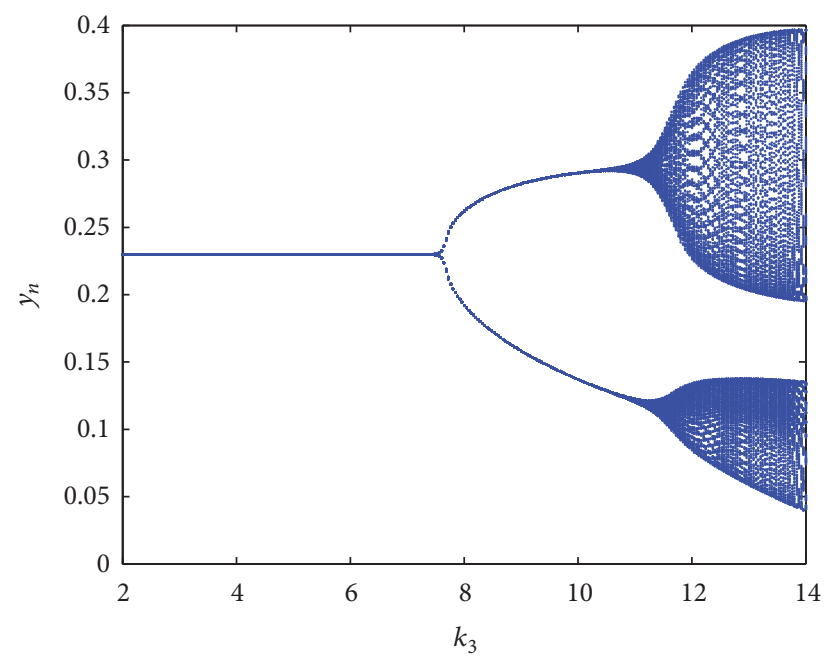

(b) Bifurcation diagram of $y_{n}$ for system (52)

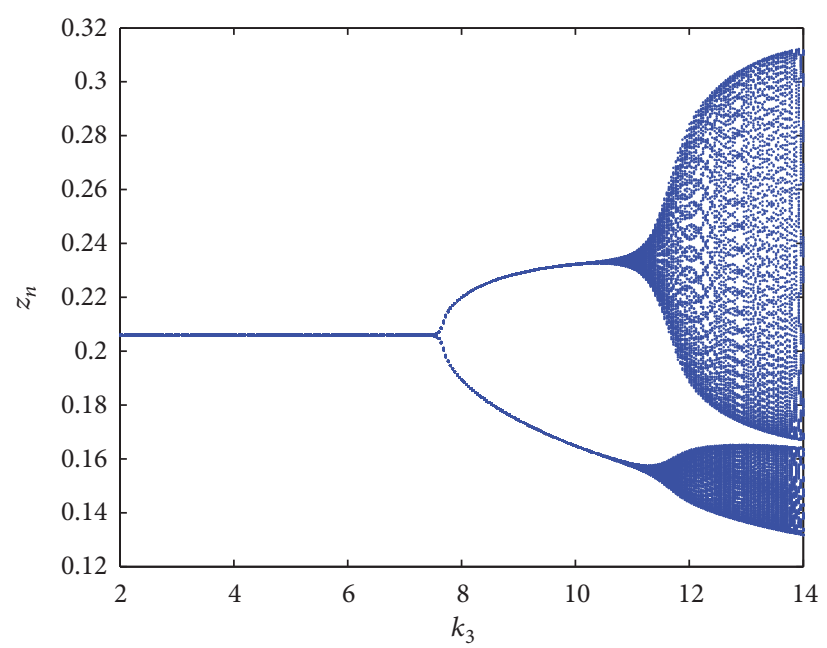

(c) Bifurcation diagram of $z_{n}$ for system (52)

FIGURE 5: Bifurcation diagrams for the controlled system (52) with $k_{1}=0.1, k_{2}=-0.1$, and $2 \leq k_{3} \leq 14$.

0.1 and $k_{2}=-0.1$, then the unique positive equilibrium point of the controlled system (52) is locally asymptotically stable if and only if $0.569236<k_{3}<7.61676$. Next, we choose $k_{1}=0.1, k_{2}=-0.1$ and selecting $k_{3} \in[2,14]$ as bifurcation parameter for the controlled system (52). Moreover, the bifurcation diagrams for the controlled system are shown in Figure 5. It can be noticed from Figure 5 that the unique positive equilibrium point $\left(x^{*}, y^{*}, z^{*}\right)=(0.363861,0.229828$, 0.205847 ) of the controlled system (52) undergoes perioddoubling bifurcation at $k_{3} \approx 7.61676$; then both periodic orbits inter the region in which these orbits undergo Neimark-Sacker bifurcation separately. Thus we succeeded to advance the Neimark-Sacker bifurcation by introducing the pole-placement control technique in this case.

Finally, choosing the parametric values $\alpha=0.3, k=0.03$, $c=0.3, \lambda=2.1, \eta=1.1, d_{1}=1.7, b_{2}=1.4, \phi=2.5, \mu=1.5$, $v=1.3$, and $\beta=3.133$ and initial conditions $x_{0}=0.37$, $y_{0}=0.23$, and $z_{0}=0.2$ in system (16), then system (16) undergoes Neimark-Sacker bifurcation for these parametric values. Then unique positive equilibrium point $\left(x^{*}, y^{*}, z^{*}\right)=$ $(0.368888,0.233346,0.207449)$ of system (16) is unstable and Figure 4(a) shows that closed invariant circle appears at $\beta=3.133$ enclosing this unstable equilibrium. For these parametric values, the controlled system (45) can be written as

$$
\begin{aligned}
& x_{n+1} \\
& =x_{n} \\
& +\frac{\rho k^{\alpha}}{\Gamma(\alpha+1)}\left[x_{n}\left(1-x_{n}\right)+\frac{\lambda c x_{n} z_{n}}{\eta+z_{n}}-\beta x_{n} y_{n}\right], \\
& y_{n+1} \\
& =y_{n} \\
& +\frac{\rho k^{\alpha}}{\Gamma(\alpha+1)}\left[\frac{\lambda d_{1} x_{n} z_{n}}{\eta+z_{n}}+\phi z_{n}-b_{2} \beta x_{n} y_{n}-\mu y_{n}\right], \\
& z_{n+1}=z_{n}+\frac{\rho k^{\alpha}}{\Gamma(\alpha+1)}\left[\beta x_{n} y_{n}-v z_{n}\right] \text {, }
\end{aligned}
$$




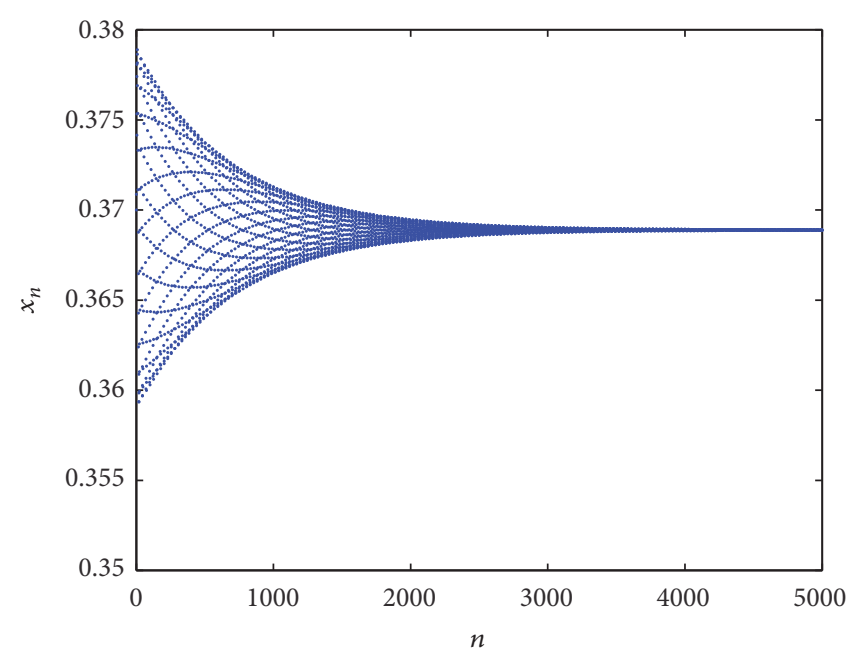

(a) Plot of $x_{n}$ for system (57)

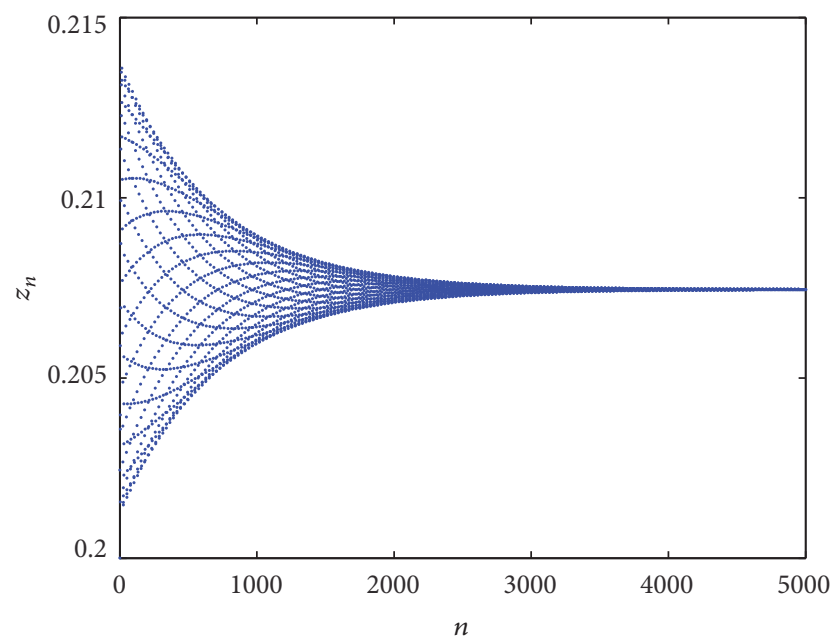

(c) Plot of $z_{n}$ for system (57)

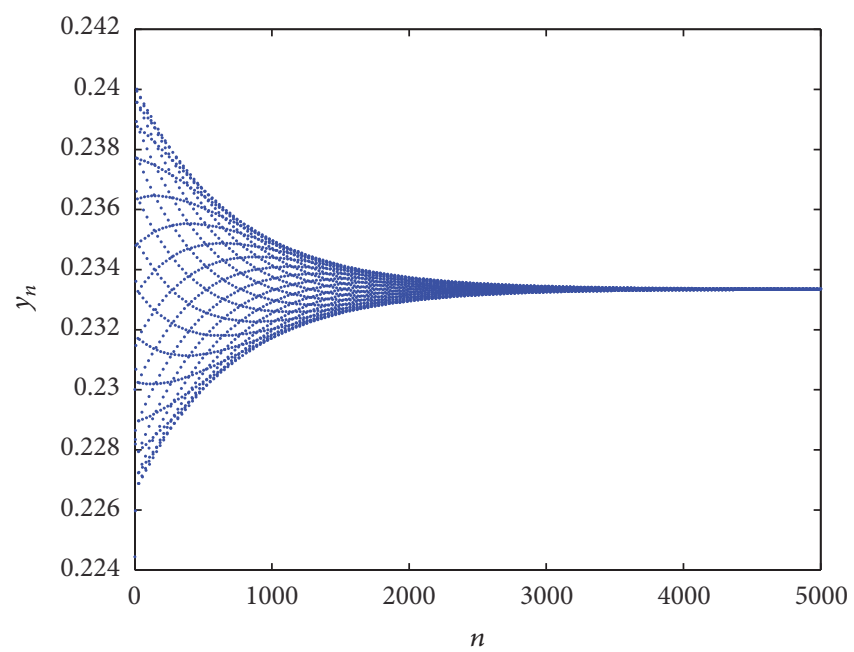

(b) Plot of $y_{n}$ for system (57)

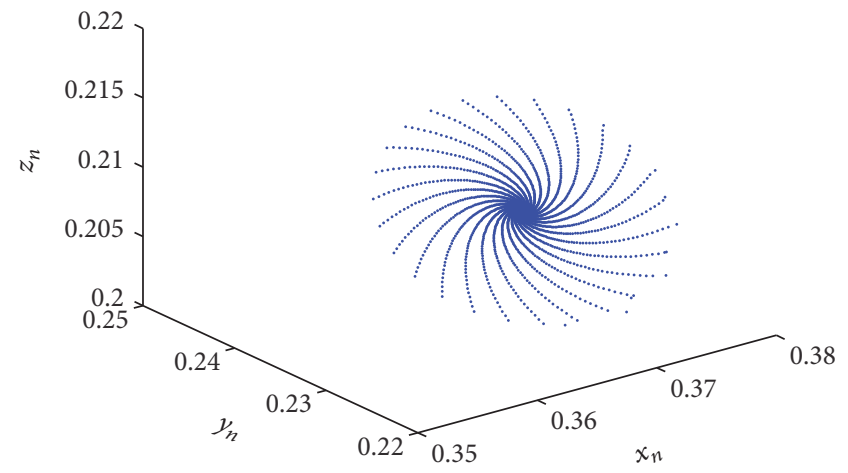

(d) Phase portrait of system (57)

FIGURE 6: Plots for the controlled system (57) with $\rho=0.95$.

where $\alpha=0.3, k=0.03, c=0.3, \lambda=2.1, \eta=1.1, d_{1}=$ $1.7, b_{2}=1.4, \phi=2.5, \mu=1.5, \nu=1.3, \beta=3.133$, and $0<\rho<1$. Then Jacobian matrix of system (57) evaluated at $\left(x^{*}, y^{*}, z^{*}\right)=(0.368888,0.233346,0.207449)$ is given by

$$
\left[\begin{array}{ccc}
1-0.143552 \rho & -0.44975 \rho & 0.0581961 \rho \\
-0.177865 \rho & 1-1.21337 \rho & 1.30265 \rho \\
0.284496 \rho & 0.44975 \rho & 1-0.505894 \rho
\end{array}\right] .
$$

The characteristic polynomial of (58) is given by

$$
\begin{aligned}
& T^{3}-(3-1.863 \rho) T^{2}+\left(3-3.726 \rho+0.178 \rho^{2}\right) T-1 \\
& +1.863 \rho-0.178 \rho^{2}+0.115 \rho^{3}=0
\end{aligned}
$$

According to the Jury condition, the roots of (58) lie in the unit open disk if and only if $0<\rho<0.996829$. Moreover, for $\rho=0.95$, the plots for the controlled system (57) are shown in Figure 6.

\section{Concluding Remarks}

We study the qualitative behavior of a 3-dimensional discretetime fractional-order plant-herbivore model, and thus we obtain the mathematical results related to existence and uniqueness of positive steady state and conditions for local asymptotic stability of positive equilibrium. In order to confirm complexity in system (16), the existence of NeimarkSacker bifurcation for positive equilibrium point is proved mathematically and numerical simulations are provided. Through numerical simulation, we show that system (16) undergoes Neimark-Sacker for wide range of bifurcation parameter $\beta$. Neimark-Sacker bifurcation is successfully controlled with two different control strategies. From our numerical investigation, it is clear that pole-placement technique based on feedback control methodology restores the stability for wide range of parameters, whereas the hybrid control based on feedback control and parameter perturbation works more effectively. Although hybrid control strategy is basically formulated for control of period-doubling bifurcation, it can 
also stabilize the unstable steady state for maximum possible range of control parameter in case of Neimark-Sacker bifurcation as well. Moreover, through numerical simulations, it is observed that the original system (16) undergoes NeimarkSacker bifurcation only and there is no chance of perioddoubling bifurcation for this system. But an application of pole-placement control strategy gives an evidence of perioddoubling bifurcation for corresponding controlled system.

\section{Conflicts of Interest}

The authors declare that they have no conflicts of interest regarding the publication of this paper.

\section{References}

[1] T. A. Revilla and F. Encinas-Viso, "Dynamical transitions in a pollination-herbivory interaction: a conflict between mutualism and antagonism," PLoS ONE, vol. 10, no. 2, Article ID e0117964, 2015.

[2] M. Caputo, "Linear models of dissipation whose Q is almost frequency independent-II," Geophysical Journal of the Royal Astronomical Society, vol. 13, no. 5, pp. 529-539, 1967.

[3] S. Rinaldi and M. Scheffer, "Geometric analysis of ecological models with slow and fast processes," Ecosystems, vol. 3, no. 6, pp. 507-521, 2000.

[4] R. P. Agarwal, Difference Equations and Inequalities, vol. 228 of Monographs and Textbooks in Pure and Applied Mathematics, Marcel Dekker, New York, NY, USA, 2nd edition, 2000.

[5] H. I. Freedman, Deterministic Mathematical Models in Population Ecology, Marcel Dekker, New York, NY, USA, 1980.

[6] B. S. Goh, Management and Analysis of Biological Populations, Elsevier Scientific, Amsterdam, The Netherlands, 1980.

[7] J. D. Murray, Mathematical Biology, vol. 19 of Biomathematics, Springer, Berlin, Germany, 1989.

[8] S. Elaydi, Discrete Chaos: With Applications in Science and Engineering, Chapman \& Hall/CRC, 2nd edition, 2008.

[9] L. J. S. Allen, An Introduction to Mathematical Biology, Prentice Hall, Upper Saddle River, NJ, USA, 2007.

[10] F. Brauer and C. Castillo-Chavez, Mathematical Models in Population Biology and Epidemiology, Springer, 2000.

[11] L. Edelstein-Keshet, Mathematical Models in Biology, vol. 46 of Classics in Applied Mathematics, SIAM, Philadelphia, Pa, USA, 2005.

[12] S. Elaydi, An Introduction to Difference Equations, Undergraduate Texts in Mathematics, Springer, New York, NY, USA, 2nd edition, 2005.

[13] Q. Din, "Global stability of beddington model," in Qualitative Theory of Dynamical Systems, pp. 1-25, Springer, Berlin, Germany, 2016.

[14] Q. Din, "Global stability and Neimark-Sacker bifurcation of a host-parasitoid model," International Journal of Systems Science, vol. 48, no. 6, pp. 1194-1202, 2017.

[15] Q. Din, "Neimark-Sacker bifurcation and chaos control in Hassell-Varley model," Journal of Difference Equations and Applications, 2017.

[16] O. Din, Ö. A. Gümüş, and H. Khalil, "Neimark-Sacker bifurcation and chaotic behaviour of a modified host-parasitoid model," Zeitschrift für Naturforschung A, vol. 72, no. 1, pp. 25-37, 2017.
[17] Q. Din, "Complexity and chaos control in a discrete-time preypredator model," Communications in Nonlinear Science and Numerical Simulation, vol. 49, pp. 113-134, 2017.

[18] A. E. Matouk, A. A. Elsadany, E. Ahmed, and H. N. Agiza, "Dynamical behavior of fractional-order Hastings-Powell food chain model and its discretization," Communications in Nonlinear Science and Numerical Simulation, vol. 27, no. 1-3, pp. 153167, 2015.

[19] J. Zhang, J. Nan, W. Du, Y. Chu, and H. Luo, "Dynamic analysis for a fractional-order autonomous chaotic system," Discrete Dynamics in Nature and Society, vol. 2016, Article ID 8712496, 13 pages, 2016.

[20] A. E. Matouk and A. A. Elsadany, "Dynamical analysis, stabilization and discretization of a chaotic fractional-order GLV model," Nonlinear Dynamics, vol. 85, no. 3, pp. 1597-1612, 2016.

[21] A. M. El-Sayed, A. Elsonbaty, A. A. Elsadany, and A. E. Matouk, "Dynamical analysis and circuit simulation of a new fractionalorder hyperchaotic system and its discretization," International Journal of Bifurcation and Chaos in Applied Sciences and Engineering, vol. 26, no. 13, 1650222, 35 pages, 2016.

[22] A. A. Elsadany and A. E. Matouk, "Dynamical behaviors of fractional-order Lotka-Volterra predator-prey model and its discretization," Journal of Applied Mathematics and Computing, vol. 49, no. 1-2, pp. 269-283, 2015.

[23] D. R. Curtiss, "Recent extensions of Descartes' rule of signs," Annals of Mathematics, vol. 19, no. 4, pp. 251-278, 1918.

[24] G. Wen, "Criterion to identify Hopf bifurcations in maps of arbitrary dimension," Physical Review E. Statistical, Nonlinear, and Soft Matter Physics, vol. 72, no. 2, Article ID 026201, 4 pages, 2005.

[25] S. Lynch, Dynamical Systems with Applications Using Mathematica, Birkhäuser, Boston, Mass, USA, 2007.

[26] F. J. Romeiras, C. Grebogi, E. Ott, and W. P. Dayawansa, "Controlling chaotic dynamical systems," Physica D. Nonlinear Phenomena, vol. 58, no. 1-4, pp. 165-192, 1992.

[27] K. Ogata, Modern Control Engineering, Prentice-Hall, Englewood, NJ, USA, 2nd edition, 1997.

[28] E. Ott, C. Grebogi, and J. A. Yorke, "Controlling chaos," Physical Review Letters, vol. 64, no. 11, pp. 1196-1199, 1990.

[29] X. S. Luo, G. R. Chen, B. H. Wang, and J. Q. Fang, "Hybrid control of period-doubling bifurcation and chaos in discrete nonlinear dynamical systems," Chaos, Solitons and Fractals, vol. 18, no. 4, pp. 775-783, 2003.

[30] L.-G. Yuan and Q.-G. Yang, "Bifurcation, invariant curve and hybrid control in a discrete-time predator-prey system," Applied Mathematical Modelling, vol. 39, no. 8, pp. 2345-2362, 2015. 


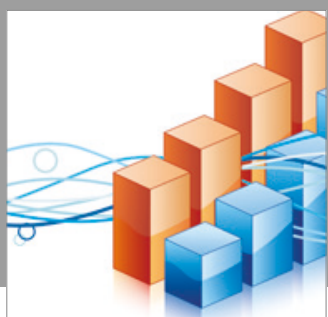

Advances in

Operations Research

vatersals

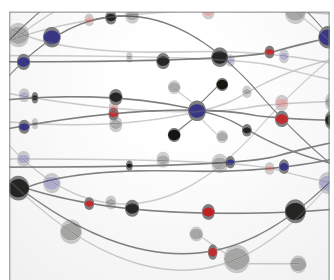

\section{The Scientific} World Journal
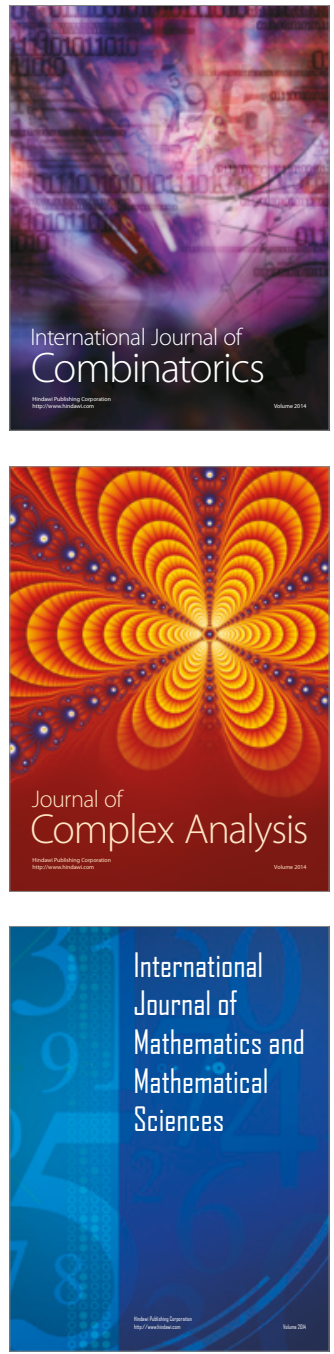
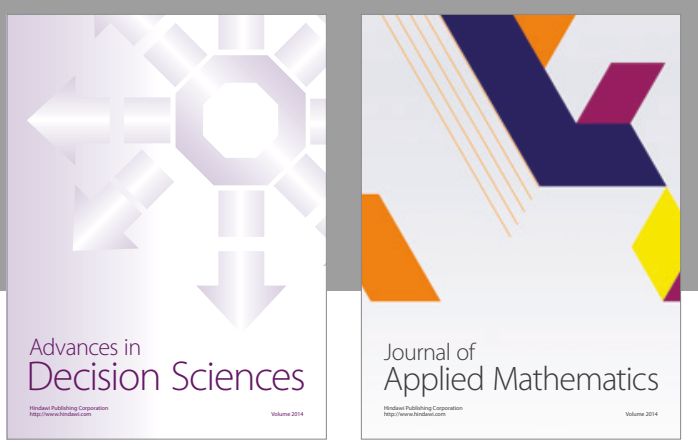

Algebra

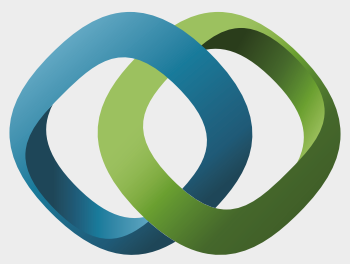

\section{Hindawi}

Submit your manuscripts at

https://www.hindawi.com
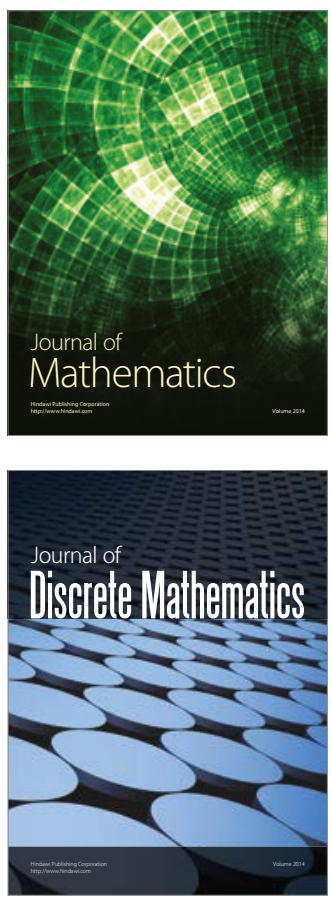

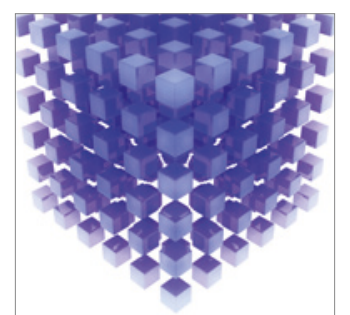

Mathematical Problems in Engineering
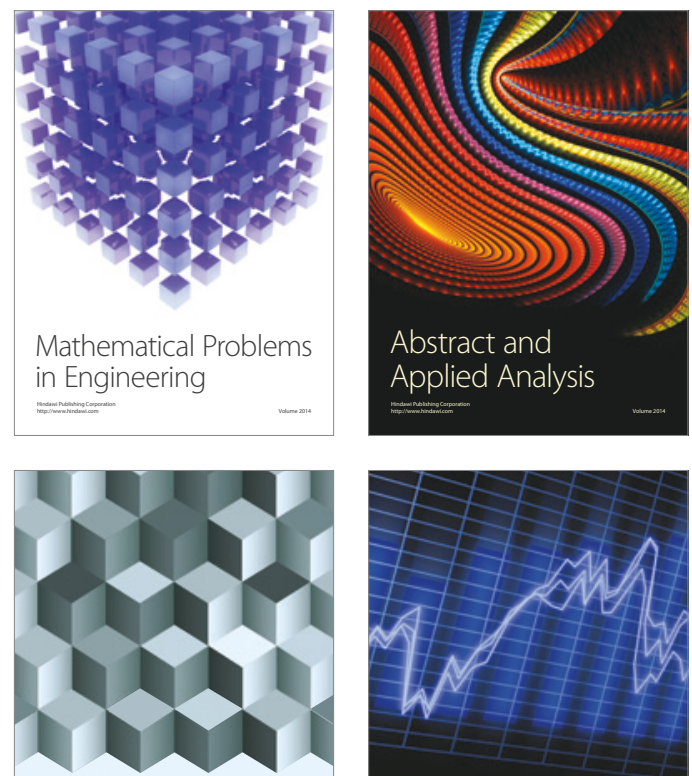

Journal of

Function Spaces

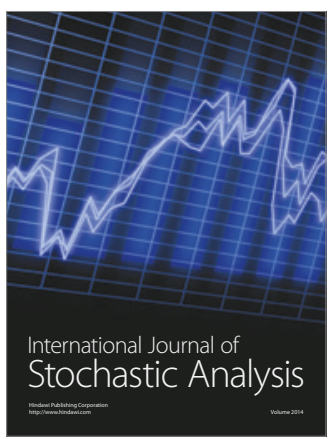

Probability and Statistics
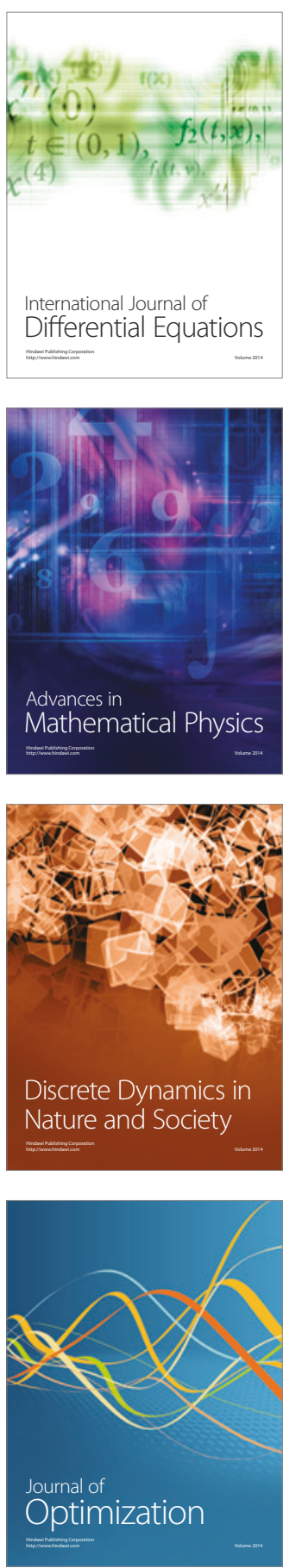